A. Cianchi $\cdot$ N. Fusco $\cdot$ F. Maggi $\cdot$ A. Pratelli

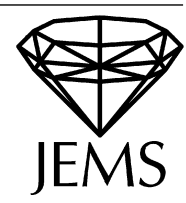

\title{
The sharp Sobolev inequality in quantitative form
}

Received November 4, 2007 and in revised form January 21, 2009

Abstract. A quantitative version of the sharp Sobolev inequality in $W^{1, p}\left(\mathbb{R}^{n}\right), 1<p<n$, is established with a remainder term involving the distance from the family of extremals.

\section{Introduction and main result}

A sharp form of the standard Sobolev inequality in $\mathbb{R}^{n}, n \geq 2$, tells us that if $1<p<n$ and $p^{*}=n p /(n-p)$, then

$$
S(p, n)\|f\|_{L^{p^{*}\left(\mathbb{R}^{n}\right)}} \leq\|\nabla f\|_{L^{p}\left(\mathbb{R}^{n}\right)}
$$

for every function $f$ from the homogeneous Sobolev space $W^{1, p}\left(\mathbb{R}^{n}\right)$ of functions $f \in$ $L^{p^{*}}\left(\mathbb{R}^{n}\right)$ such that $\nabla f \in L^{p}\left(\mathbb{R}^{n}\right)$. Here

$$
S(p, n)=\sqrt{\pi} n^{1 / p}\left(\frac{n-p}{p-1}\right)^{(p-1) / p}\left(\frac{\Gamma(n / p) \Gamma(1+n-n / p)}{\Gamma(1+n / 2) \Gamma(n)}\right)^{1 / n}
$$

is the best possible constant in 11.1 , and $\|\nabla f\|_{L^{p}\left(\mathbb{R}^{n}\right)}$ stands for the $L^{p}\left(\mathbb{R}^{n}\right)$ norm of the length $|\nabla f|$ of the gradient $([\mathrm{Au}, \mid \mathrm{Ta}])$. A family of extremals in $(1.1)$ is given by the functions $g_{a, b, x_{0}}: \mathbb{R}^{n} \rightarrow[0, \infty)$ defined as

$$
g_{a, b, x_{0}}(x)=\frac{a}{\left(1+b\left|x-x_{0}\right|^{p^{\prime}}\right)^{(n-p) / p}} \quad \text { for } x \in \mathbb{R}^{n}
$$

for some $a \neq 0, b>0, x_{0} \in \mathbb{R}^{n}$. Here, $p^{\prime}=p /(p-1)$, the Hölder conjugate of $p$. In fact, as pointed out by the recent contribution $[\mathrm{CNV}]$, functions having the form 1.2$]$ are the only ones attaining equality in 1.1 for every $p \in(1, n)$. Incidentally, note that,

A. Cianchi: Dipartimento di Matematica e Applicazioni per l'Architettura, Piazza Ghiberti 27, 50122 Firenze, Italy; e-mail: cianchi@unifi.it

N. Fusco: Dipartimento di Matematica ed Applicazioni, via Cintia, 80126 Napoli, Italy; e-mail: nicola.fusco@unina.it

F. Maggi: Dipartimento di Matematica, viale Morgagni 67/A, 50134 Firenze, Italy; e-mail:maggi@math.unifi.it

A. Pratelli: Dipartimento di Matematica, via Ferrata 1, 27100 Pavia, Italy; e-mail: aldo.pratelli@unipv.it 
when $p=2$, the classical result of [GNN], applied to the Euler equation of the functional $\|\nabla f\|_{L^{2}\left(\mathbb{R}^{n}\right)} /\|f\|_{L^{2^{\star}}\left(\mathbb{R}^{n}\right)}$, can alternatively be used to derive this characterization of the extremals in 1.1 .

The objective of the present paper is to strengthen inequality 1.1 by an additional term on the left-hand side which accounts for the deviation of $f$ from extremals. More precisely, we establish a quantitative version of inequality (1.1), with a remainder term depending on the (normalized) distance of $f$ from the family of extremals 1.2 given by

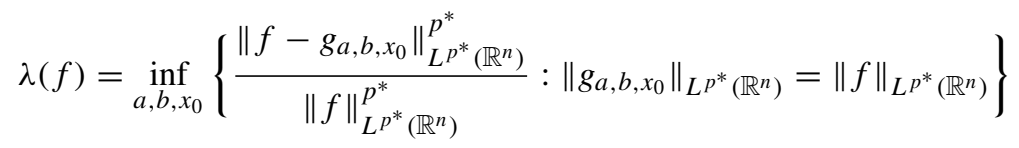

if $f \neq 0$, and $\lambda(0)=0$.

Theorem 1. Let $n \geq 2$ and let $1<p<n$. Then there exist positive constants $\alpha$ and $\kappa$, depending only on $p$ and $n$, such that

$$
S(p, n)\|f\|_{L^{p^{*}\left(\mathbb{R}^{n}\right)}}\left(1+\kappa \lambda(f)^{\alpha}\right) \leq\|\nabla f\|_{L^{p}\left(\mathbb{R}^{n}\right)}
$$

for every $f \in W^{1, p}\left(\mathbb{R}^{n}\right)$.

In analogy with the terminology of [Fu, Ha, HHW, FMP1, FMP2], we will refer to $\lambda(f)$ as the asymmetry of $f$. Notice that one could alternatively consider the quantity defined as

$$
d(f)=\inf _{a, b, x_{0}} \frac{\left\|f-g_{a, b, x_{0}}\right\|_{L^{p^{*}}\left(\mathbb{R}^{n}\right)}}{\|f\|_{L^{p^{*}}\left(\mathbb{R}^{n}\right)}}
$$

if $f \neq 0$, and $d(0)=0$. It is obvious that $d(f) \leq \lambda(f)^{1 / p^{*}}$; on the other hand, one can check that $\lambda(f)^{1 / p^{*}} \leq 2 d(f)$. Therefore, inequality $(1.4)$ is equivalent to

$$
S(p, n)\|f\|_{L^{p^{*}\left(\mathbb{R}^{n}\right)}}\left(1+\kappa d(f)^{\theta}\right) \leq\|\nabla f\|_{L^{p}\left(\mathbb{R}^{n}\right)}
$$

with $\theta=p^{*} \alpha$, up to changing the value of $\kappa$.

Inequality 1.5 gives a positive answer to a question raised by Brezis and Lieb in $[\overline{\mathrm{BL}}]$, which has been settled in $[\overline{\mathrm{BE}}]$ in the special case when $p=2$ in the even stronger form with $\left\|f-g_{a, b, x_{0}}\right\|_{L^{2^{\star}}\left(\mathbb{R}^{n}\right)}$ replaced by $\left\|\nabla f-\nabla g_{a, b, x_{0}}\right\|_{L^{2}\left(\mathbb{R}^{n}\right)}$ in 1.3 . The method of [BE] heavily rests upon the Hilbert space structure of $W^{1,2}\left(\mathbb{R}^{n}\right)$ and on the eigenvalue properties of a weighted Laplacian in $\mathbb{R}^{n}$. Such an approach, which has been employed to deal with other related problems involving Sobolev spaces endowed with a Hilbert space structure ([Lo, BWW]), does not seem suitable for extensions to the general case where $p \neq 2$. Following the lines traced in $[\mathrm{Au}]$ and $[\mathrm{Ta}]$, we have instead to resort to certain methods of geometric flavor, exploiting such tools as isoperimetric inequalities and symmetrizations. Developments of these results led to quantitative forms of isoperimetric ([Fu, Ha, FMP1] $)$, isocapacitary ([HHW], FMP3]) and Sobolev inequalities ([Ci1, FMP2, Ci2]) in the spirit of (1.4).

To be more specific, the proof of Theorem 1 basically consists of three steps, each step amounting to an extension of inequality (1.4) to a broader class of functions. After 
starting with spherically symmetric functions, we proceed with $n$-symmetric functions, i.e. functions which are symmetric about $n$ orthogonal hyperplanes, and we eventually conclude with arbitrary Sobolev functions. This strategy can be clarified by the following considerations.

The operation of spherically symmetric rearrangement, which associates with any nonnegative function $f \in W^{1, p}\left(\mathbb{R}^{n}\right)$ the spherically symmetric equidistributed function $f^{\star} \in W^{1, p}\left(\mathbb{R}^{n}\right)($ see 3.2$)$ ), satisfies

$$
\left\|f^{\star}\right\|_{L^{p^{*}\left(\mathbb{R}^{n}\right)}}=\|f\|_{L^{p^{*}\left(\mathbb{R}^{n}\right)}}
$$

and

$$
\left\|\nabla f^{\star}\right\|_{L^{p}\left(\mathbb{R}^{n}\right)} \leq\|\nabla f\|_{L^{p}\left(\mathbb{R}^{n}\right)}
$$

([BZ, $\overline{\mathrm{Ka}}, \mathrm{Ta}])$. As a consequence,

$$
\left\|\nabla f^{\star}\right\|_{L^{p}\left(\mathbb{R}^{n}\right)}-S(p, n)\left\|f^{\star}\right\|_{L^{p}\left(\mathbb{R}^{n}\right)} \leq\|\nabla f\|_{L^{p}\left(\mathbb{R}^{n}\right)}-S(p, n)\|f\|_{L^{p^{*}}\left(\mathbb{R}^{n}\right)}
$$

and

$$
\|\nabla f\|_{L^{p}\left(\mathbb{R}^{n}\right)}-\left\|\nabla f^{\star}\right\|_{L^{p}\left(\mathbb{R}^{n}\right)} \leq\|\nabla f\|_{L^{p}\left(\mathbb{R}^{n}\right)}-S(p, n)\|f\|_{L^{p^{*}}\left(\mathbb{R}^{n}\right)}
$$

for every $f \in W^{1, p}\left(\mathbb{R}^{n}\right)$. In view of $(1.7)$ and 1.8 , the underlying idea in the proof of inequality (1.4) is to split the problem: first, establishing the inequality in the class of spherically symmetric functions; second, estimating the $L^{p^{*}}$ distance of $f$ from (a suitable translate of) $f^{\star}$ in terms of $\|\nabla f\|_{L^{p}\left(\mathbb{R}^{n}\right)}-\left\|\nabla f^{\star}\right\|_{L^{p}\left(\mathbb{R}^{n}\right)}$.

Even in the special class of spherically symmetric functions, the derivation of (1.4) is not straightforward. Actually, standard proofs of the one-dimensional Bliss inequality, to which (1.1) reduces when restricted to spherically symmetric functions, do not seem suitable for modifications yielding stability results. A more flexible approach to the relevant one-dimensional inequality, which can be successfully extended to provide a quantitative version, follows instead on specializing a mass transportation technique employed in [CNV] (see also [LYZ]). The resulting estimate, whose proof also requires a sharp version of a trace Sobolev inequality from [MV], is contained in Theorem 2, and settles the first of the two steps outlined above.

Major problems arise in the attempt at estimating the asymmetry of $f$ in terms of the left-hand side of (1.8). Indeed, this is just impossible, without additional assumptions on $f$, as demonstrated by simple examples where $\|\nabla f\|_{L^{p}\left(\mathbb{R}^{n}\right)}$ almost agrees with $\left\|\nabla f^{\star}\right\|_{L^{p}\left(\mathbb{R}^{n}\right)}$, without $f$ being close to any translate of $f^{\star}$. The presence of plateaus in the graph of $f$, or more generally, of large sets where $\left|\nabla f^{\star}\right|$ is small, is responsible for this phenomenon (see e.g. [BZ, $\mathrm{CF} 2]$ ). A key observation to overcome this obstacle is that a bound for the distance of $f$ from $f^{\star}$ via $\|\nabla f\|_{L^{p}\left(\mathbb{R}^{n}\right)}-\left\|\nabla f^{\star}\right\|_{L^{p}\left(\mathbb{R}^{n}\right)}$ can be restored if $f$ is already known to enjoy certain partial symmetry properties. It is at this stage that the class of $n$-symmetric functions comes into play. Indeed, on the one hand, the distance of $f$ from $f^{\star}$ can actually be estimated by $\|\nabla f\|_{L^{p}\left(\mathbb{R}^{n}\right)}-\left\|\nabla f^{\star}\right\|_{L^{p}\left(\mathbb{R}^{n}\right)}$ if $f$ is a priori assumed to be $n$-symmetric (Theorem 3), thus enabling us to establish (1.4) in this class of functions (Corollary 4). On the other hand, any function $f \in W^{1, p}\left(\mathbb{R}^{n}\right)$ can be replaced, through a careful construction exploiting reflection arguments, by a suitable $n$-symmetric 
function in such a way that $\|\nabla f\|_{L^{p}\left(\mathbb{R}^{n}\right)}-S(p, n)\|f\|_{L^{p^{*}}\left(\mathbb{R}^{n}\right)}$ and $\lambda(f)$ do not increase and decrease, respectively, too much (Theorem 6). This fact, combined with the former step, easily leads to the conclusion of Theorem 1 1 . Let us emphasize that the reduction to $n$-symmetric functions, although related to a similar construction employed in [FMP1, FMP2], entails the overcoming of serious new obstacles in the present setting, mainly due to the nonlinear growth of the functional $\|\nabla f\|_{L^{p}\left(\mathbb{R}^{n}\right)}^{p}$.

We conclude this section by noting that, in view of the results of [BE] and [FMP2], the question arises of the optimal exponent $\alpha$ in equality (1.4). Furthermore, the result of $[\mathrm{BE}]$ also leaves open the problem of whether the distance of $f$ from the family of extremals in $L^{p^{*}}\left(\mathbb{R}^{n}\right)$ can be replaced by the distance in $W^{1, p}\left(\mathbb{R}^{n}\right)$ in Theorem 1

\section{A quantitative Bliss inequality}

In the present section, Theorem 1 will be established in the special class of spherically symmetric functions. Notice that the Sobolev inequality (1.1), restricted to this class of functions, is equivalent to the one-dimensional Bliss inequality

$$
S(p, n)\left(n \omega_{n} \int_{0}^{\infty} u(r)^{p^{*}} r^{n-1} d r\right)^{1 / p^{*}} \leq\left(n \omega_{n} \int_{0}^{\infty}\left(-u^{\prime}(r)\right)^{p} r^{n-1} d r\right)^{1 / p}
$$

for every decreasing, locally absolutely continuous function $u:[0, \infty) \rightarrow[0, \infty)$, where $\omega_{n}$ is the measure of the unit ball in $\mathbb{R}^{n}$. The extremals in 2.1] have the form

$$
v_{a, b}(r)=\frac{a}{\left(1+b r p^{p^{\prime}}\right)^{(n-p) / p}} \quad \text { for } r \geq 0,
$$

for some $a>0, b>0([\mathrm{~B} 1, \mathrm{CNV}, \mathrm{LYZ}, \mathrm{Ta}])$. Thus, on setting, with a slight abuse of notation,

$$
\begin{aligned}
& \lambda(u)=\inf \left\{\frac{\int_{0}^{\infty}\left|u(r)-v_{a, b}(r)\right|^{p^{*}} r^{n-1} d r}{\int_{0}^{\infty} u(r)^{p^{*}} r^{n-1} d r}:\right. \\
&\left.\int_{0}^{\infty} v_{a, b}(r)^{p^{*}} r^{n-1} d r=\int_{0}^{\infty} u(r)^{p^{*}} r^{n-1} d r, a, b>0\right\},
\end{aligned}
$$

Theorem 1 for spherically symmetric functions is equivalent to the following quantitative Bliss inequality.

Theorem 2. Let $n \geq 2$ and let $1<p<n$. Then there exist constants $\beta$ and $\kappa$ such that

$$
\begin{aligned}
S(p, n)\left(n \omega_{n} \int_{0}^{\infty} u(r)^{p^{*}} r^{n-1} d r\right)^{1 / p^{*}} & \left(1+\kappa \lambda(u)^{\beta}\right) \\
\leq & \left(n \omega_{n} \int_{0}^{\infty}\left(-u^{\prime}(r)\right)^{p} r^{n-1} d r\right)^{1 / p}
\end{aligned}
$$

for every decreasing, locally absolutely continuous function $u:[0, \infty) \rightarrow[0, \infty)$. 
In the proof of Theorem 2, we shall make use of the notation

$$
\delta(u)=\frac{\left(n \omega_{n} \int_{0}^{\infty}\left(-u^{\prime}(r)\right)^{p} r^{n-1} d r\right)^{1 / p}}{S(p, n)\left(n \omega_{n} \int_{0}^{\infty} u(r)^{p^{*}} r^{n-1} d r\right)^{1 / p^{*}}}-1,
$$

so that $(2.3)$ can be rewritten as

$$
\lambda(u) \leq C \delta(u)^{1 / \beta},
$$

where $C=\kappa^{-1 / \beta}$.

Proof of Theorem 2 Approximation, rescaling and normalization arguments allow us to assume that $u$ is continuously differentiable, with support equal to $[0,1]$, and that

$$
n \omega_{n} \int_{0}^{\infty} u(r)^{p^{*}} r^{n-1} d r=1 .
$$

Moreover, for the time being, we assume that

$$
\delta(u) \leq \varepsilon(p, n)
$$

for some positive constant $\varepsilon(p, n)<1$, to be chosen later. Let us set

$$
v(r)=v_{a, 1}(r) \quad \text { for } r>0,
$$

where $a$ is such that

$$
n \omega_{n} \int_{0}^{\infty} v(r)^{p^{*}} r^{n-1} d r=1
$$

Owing to 2.6) and (2.7), the equation

$$
\int_{0}^{r} u(s)^{p^{*}} s^{n-1} d s=\int_{0}^{T(r)} v(s)^{p^{*}} s^{n-1} d s
$$

implicitly defines a strictly increasing function $T:[0,1) \rightarrow[0, \infty)$ such that $T \in$ $C^{1}(0,1), T(0)=0, \lim _{r \rightarrow 1^{-}} T(r)=\infty$, and

$$
u(r)^{p^{*}}=v(T(r))^{p^{*}} M(r)^{n-1} T^{\prime}(r) \quad \text { for } r \in(0,1),
$$

where $M:(0,1) \rightarrow(0, \infty)$ is given by

$$
M(r)=\frac{T(r)}{r} \quad \text { for } r \in(0,1) .
$$

In particular, equation 2.9 ) entails that

$$
\int_{0}^{1} h(T(r)) u(r)^{p^{*}} r^{n-1} d r=\int_{0}^{\infty} h(r) v(r)^{p^{*}} r^{n-1} d r
$$

for every Borel function $h:[0, \infty) \rightarrow[0, \infty]$. In the terminology of the theory of mass transportation, by which the present proof is inspired, the function $T$ can be regarded as a transport map carrying the density $u(r)^{p^{*}} r^{n-1}$ into $v(r)^{p^{*}} r^{n-1}$. 
Notice that when $T(r)=k r$ for some $k>0$, one has $u(r)=k^{(n-p) / p} v(k r)$, that is, $u$ is an extremal function in the Bliss inequality (2.1). Thus, our plan is to show that, if $\delta(u)$ is small, then an interval $\left[r_{1}, r_{2}\right] \subseteq[0,1]$ can be chosen in such a way that $T(r)$ is close to some linear function $k r$ for $r \in\left[r_{1}, r_{2}\right]$, and simultaneously the integral of $u(r)^{p^{*}} r^{n-1}$ outside $\left[r_{1}, r_{2}\right]$ is small. These facts will enable us to conclude that $u$ is close to $k^{(n-p) / p} v(k r)$ in $L^{p^{*}}\left(r^{n-1} d r\right)$.

For ease of presentation, we accomplish the proof in several steps.

Step I. Mass transportation proof of Bliss inequality. We begin by giving a proof of the Bliss inequality relying on the mass transportation approach of [CNV] (see also [LYZ]). Set $p^{\sharp}=p(n-1) /(n-p)$, the optimal exponent in the trace inequality in $\mathbb{R}^{n}$. Owing to 2.10 and 2.9 we have

$$
\begin{aligned}
\int_{0}^{\infty} v(r)^{p^{\sharp}} r^{n-1} d r & =\int_{0}^{\infty} v(r)^{-p^{*} / n} v(r)^{p^{*}} r^{n-1} d r=\int_{0}^{1} v(T(r))^{-p^{*} / n} u(r)^{p^{*}} r^{n-1} d r \\
& =\int_{0}^{1}\left(\frac{M(r)^{n-1} T^{\prime}(r)}{u(r)^{p^{*}}}\right)^{1 / n} u(r)^{p^{*}} r^{n-1} d r \\
& =\int_{0}^{1} M(r)^{1 / n^{\prime}} T^{\prime}(r)^{1 / n} u(r)^{p^{\sharp}} r^{n-1} d r .
\end{aligned}
$$

By Young's inequality,

$$
\begin{aligned}
\int_{0}^{1} M(r)^{1 / n^{\prime}} T^{\prime}(r)^{1 / n} u(r)^{p^{\sharp}} r^{n-1} d r & \leq \frac{1}{n} \int_{0}^{1}\left(T^{\prime}(r)+(n-1) M(r)\right) u(r)^{p^{\sharp}} r^{n-1} d r \\
& =\frac{1}{n} \int_{0}^{1}\left(r^{n-1} T(r)\right)^{\prime} u(r)^{p^{\sharp}} d r \\
& =\frac{p^{\sharp}}{n} \int_{0}^{1} T(r)\left(-u^{\prime}(r)\right) u(r)^{p^{\sharp}-1} r^{n-1} d r .
\end{aligned}
$$

The last equality can be justified as follows. By Hölder's inequality and 2.10,

$$
\begin{aligned}
\int_{0}^{1} T(r)(- & \left.u^{\prime}(r)\right) u(r)^{p^{\sharp}-1} r^{n-1} d r \\
& \leq\left(\int_{0}^{1}\left(-u^{\prime}(r)\right)^{p} r^{n-1} d r\right)^{1 / p}\left(\int_{0}^{1} T(r)^{p^{\prime}} u(r)^{p^{*}} r^{n-1} d r\right)^{1 / p^{\prime}} \\
& =\left(\int_{0}^{1}\left(-u^{\prime}(r)\right)^{p} r^{n-1} d r\right)^{1 / p}\left(\int_{0}^{\infty} v(r)^{p^{*}} r^{p^{\prime}+n-1} d r\right)^{1 / p^{\prime}}
\end{aligned}
$$

In particular,

$$
\int_{0}^{1} T(r)\left(-u^{\prime}(r)\right) u(r)^{p^{\sharp}-1} r^{n-1} d r<\infty .
$$


Since $u$ is bounded, an integration by parts yields

$$
\begin{aligned}
\int_{0}^{R}\left(r^{n-1} T(r)\right)^{\prime} u(r)^{p^{\sharp}} d r= & R^{n-1} T(R) u(R)^{p^{\sharp}} \\
& +p^{\sharp} \int_{0}^{R} T(r)\left(-u^{\prime}(r)\right) u(r)^{p^{\sharp}-1} r^{n-1} d r
\end{aligned}
$$

for $0<R<1$. Observe now that, since $u(1)=0$,

$$
\begin{aligned}
\int_{R}^{1} T(r)\left(-u^{\prime}(r)\right) u(r)^{p^{\sharp}-1} r^{n-1} d r & \geq T(R) R^{n-1} \int_{R}^{1}-u^{\prime}(r) u(r)^{p^{\sharp}-1} d r \\
& =\frac{T(R) R^{n-1} u(R)^{p^{\sharp}}}{p^{\sharp}} .
\end{aligned}
$$

Hence by 2.14 it follows that $T(R) u(R)^{p^{\sharp}} \rightarrow 0$ as $R \rightarrow 1$, so that the last equality in 2.12 follows on passing to the limit in 2.15].

Now, define $\zeta:[0, \infty) \rightarrow[0, \infty)$ as

$$
\zeta(t)=t+(n-1)-n t^{1 / n} \quad \text { for } t \geq 0,
$$

and set

$$
C_{0}=p^{\sharp}\left(\int_{0}^{\infty} v(r)^{p^{*}} r^{p^{\prime}+n-1} d r\right)^{1 / p^{\prime}},
$$

a constant depending only on $p$ and $n$. Formulas 2.11-2.13 entail that

$$
\begin{aligned}
\int_{0}^{1} \zeta\left(\frac{T^{\prime}(r)}{M(r)}\right) M(r) u(r)^{p^{\sharp}} r^{n-1} d r \leq & C_{0}\left(\int_{0}^{1}\left(-u^{\prime}(r)\right)^{p} r^{n-1} d r\right)^{1 / p} \\
& -n \int_{0}^{\infty} v^{p^{\sharp}} r^{n-1} d r .
\end{aligned}
$$

One can easily verify that

$$
C_{0}\left(\int_{0}^{\infty}\left(-v^{\prime}(r)\right)^{p} r^{n-1} d r\right)^{1 / p}=n \int_{0}^{\infty} v^{p^{\sharp}} r^{n-1} d r .
$$

Consequently, recalling (2.7), a direct calculation shows that

$$
n \int_{0}^{\infty} v(r)^{p^{\sharp}} r^{n-1} d r=\frac{C_{0} S(p, n)}{\left(n \omega_{n}\right)^{1 / p}}
$$

In conclusion, 2.16 tells us that

$$
\int_{0}^{1} \zeta\left(\frac{T^{\prime}(r)}{M(r)}\right) M(r) u(r)^{p^{\sharp}} r^{n-1} d r \leq \frac{C_{0} S(p, n)}{\left(n \omega_{n}\right)^{1 / p}} \delta(u)
$$

for every $u$ as in the statement. Notice that if $\delta(u)=0$ then 2.17 gives $T^{\prime}(r) / M(r)=1$ for all $r \in(0,1)$; hence $T(r)=k r$ and as observed above this implies that $u$ is as in (2.2). 
This observation was a crucial point in [CNV]. In our case, instead, we have to extract a quantitative information from 2.17) by proving that if $\delta(u)$ is small then $T(r)$ is close to a suitable linear function of $r$.

Step II. A lower bound for $u(r)^{p^{\sharp}} r^{n-1}$. We now prove a bound for $u(r)^{p^{\sharp}} r^{n-1}$ from below in a suitable subinterval of $(0,1)$, and we combine it with 2.9 ) to derive an integral estimate on such intervals involving $T$ and $T^{\prime}$. A key ingredient here is a trace inequality from [MV, Theorem 1.3], which, in the one-dimensional case, tells us that

$$
\begin{aligned}
\left(n \omega_{n} \int_{0}^{r} u(s)^{p^{*}} s^{n-1} d s\right)^{p / p^{*}} & \\
& \leq \frac{n \omega_{n}}{S(p, n)^{p}} \int_{0}^{r}\left(-u^{\prime}(s)\right)^{p} s^{n-1} d s+C_{1}\left(u(r)^{p^{\sharp}} r^{n-1}\right)^{p / p^{\sharp}}
\end{aligned}
$$

and

$$
\begin{aligned}
\left(n \omega_{n} \int_{r}^{1} u(s)^{p^{*}} s^{n-1} d s\right)^{p / p^{*}} & \\
& \leq \frac{n \omega_{n}}{S(p, n)^{p}} \int_{r}^{1}\left(-u^{\prime}(s)\right)^{p} s^{n-1} d s+C_{1}\left(u(r)^{p^{\sharp}} r^{n-1}\right)^{p / p^{\sharp}}
\end{aligned}
$$

for every $0<r<1$, for some constant $C_{1}>0$. Set

$$
\gamma(r)=n \omega_{n} \int_{0}^{r} u(s)^{p^{*}} s^{n-1} d s \quad \text { for } r \in[0,1] .
$$

Adding up inequalities 2.18) and 2.19) implies that

$$
\begin{aligned}
\gamma(r)^{p / p^{*}}+(1-\gamma(r))^{p / p^{*}} & \leq(1+\delta(u))^{p}+2 C_{1}\left(u(r)^{p^{\sharp}} r^{n-1}\right)^{p / p^{\sharp}} \\
& \leq 1+C_{2} \delta(u)+2 C_{1}\left(u(r)^{p^{\sharp}} r^{n-1}\right)^{p / p^{\sharp}}
\end{aligned}
$$

for some positive constant $C_{2}$. Notice that the second inequality holds owing to 2.6. On setting

$$
\psi(t)=t^{p / p^{*}}+(1-t)^{p / p^{*}}-1 \quad \text { for } t \in[0,1],
$$

inequality 2.20$)$ reads

$$
\left(\psi(\gamma(r))-C_{2} \delta(u)\right)^{p^{\sharp} / p} \leq C_{3} u(r)^{p^{\sharp}} r^{n-1} \quad \text { for } r \in[0,1],
$$

with $C_{3}=\left(2 C_{1}\right)^{p^{\sharp} / p}$. It is easily seen that there exists a positive constant $C_{4}$ such that, if $0<\varepsilon<1 / C_{4}$, then

$$
\psi(t) \geq 3 \varepsilon \quad \text { for } t \in\left[(4 \varepsilon)^{p^{*} / p}, 1-(4 \varepsilon)^{p^{*} / p}\right] .
$$

Hence, given any $\varepsilon \in\left(0,1 / C_{4}\right)$, on denoting by $r_{1}$ and $r_{2}$ the positive numbers satisfying

$$
\gamma\left(r_{1}\right)=(4 \varepsilon)^{p^{*} / p}, \quad \gamma\left(r_{2}\right)=1-(4 \varepsilon)^{p^{*} / p},
$$


and assuming that

$$
\delta(u) \leq \varepsilon / C_{2},
$$

we get

$$
(2 \varepsilon)^{p^{\sharp} / p} \leq C_{3} u(r)^{p^{\sharp}} r^{n-1} \quad \text { for } r \in\left[r_{1}, r_{2}\right] .
$$

On the other hand, owing to 2.22) and 2.24, inequality 2.21) entails that

$$
(\psi(\gamma(r)) / 2)^{p^{\sharp} / p} \leq C_{3} u(r)^{p^{\sharp}} r^{n-1} \quad \text { for } r \in\left[r_{1}, r_{2}\right] .
$$

Since $\gamma^{\prime}(r)=n \omega_{n} u(r)^{p^{*}} r^{n-1}$ for $r>0$, we infer from 2.26) and 2.9) that

$$
\begin{aligned}
C_{5} \frac{\gamma^{\prime}(r)}{\psi(\gamma(r))^{p^{\sharp} / p}} & \geq u(r)^{p^{*}-p^{\sharp}}=u(r)^{p^{*} / n} \\
& =v(T(r))^{p^{*} / n} M(r)^{1 / n^{\prime}} T^{\prime}(r)^{1 / n} \quad \text { for every } r \in\left[r_{1}, r_{2}\right],
\end{aligned}
$$

for some positive constant $C_{5}$. Hence,

$$
\int_{r_{1}}^{r_{2}} v(T(r))^{p^{*} / n} M(r)^{1 / n^{\prime}} T^{\prime}(r)^{1 / n} d r \leq C_{6}
$$

for some constant $C_{6}$.

Step III. An integral bound for $\left|T^{\prime}-M\right|$. The task of the present step is to provide an estimate for $\int_{r_{1}}^{r_{2}}\left|T^{\prime}(r)-M(r)\right| d r$. Our starting point is the inequality

$$
\int_{r_{1}}^{r_{2}} M(r) \zeta\left(\frac{T^{\prime}(r)}{M(r)}\right) d r \leq C_{7} \frac{\delta(u)}{\varepsilon^{p^{\sharp} / p}},
$$

which follows from 2.17) and 2.25) and holds for some positive constant $C_{7}$. Since $\zeta^{\prime}(1)=\zeta(1)=0$ and $\zeta^{\prime \prime}(t)=\left(1 / n^{\prime}\right) t^{-2+1 / n}$, a decreasing function, by Taylor's formula we have

$$
\zeta(t) \geq \frac{1}{2 n^{\prime}} \min \left\{1, t^{-2+1 / n}\right\}(t-1)^{2} \quad \text { for } t \geq 0 .
$$

Thus, inequality 2.28 tells us that

$$
2 C_{7} n^{\prime} \frac{\delta(u)}{\varepsilon^{p^{\sharp} / p}} \geq \int_{r_{1}}^{r_{2}} \frac{\left(T^{\prime}(r)-M(r)\right)^{2}}{M(r)} \min \left\{1,\left(\frac{M(r)}{T^{\prime}(r)}\right)^{2-1 / n}\right\} d r .
$$

Define

$$
I=\left\{r \in\left[r_{1}, r_{2}\right]: T^{\prime}(r) \leq M(r)\right\}, \quad J=\left[r_{1}, r_{2}\right] \backslash I .
$$

By 2.29), Hölder's inequality and 2.27),

$$
\begin{aligned}
2 C_{7} n^{\prime} \frac{\delta(u)}{\varepsilon^{p^{\sharp} / p}} & \geq \int_{J} \frac{\left(T^{\prime}(r)-M(r)\right)^{2}}{T^{\prime}(r)^{2} v(T(r))^{p^{*} / n}} v(T(r))^{p^{*} / n} T^{\prime}(r)^{1 / n} M(r)^{1 / n^{\prime}} d r \\
& \geq \frac{1}{C_{6}}\left(\int_{J} \frac{\left|T^{\prime}(r)-M(r)\right|}{T^{\prime}(r) v(T(r))^{p^{*} / 2 n}} v(T(r))^{p^{*} / n} T^{\prime}(r)^{1 / n} M(r)^{1 / n^{\prime}} d r\right)^{2} \\
& =\frac{1}{C_{6}}\left(\int_{J}\left|T^{\prime}(r)-M(r)\right| v(T(r))^{p^{*} / 2 n}\left(\frac{M(r)}{T^{\prime}(r)}\right)^{1 / n^{\prime}} d r\right)^{2} .
\end{aligned}
$$


From 2.8 we deduce that

$$
a^{p^{*}} \frac{T(r)^{n}}{n} \geq \int_{0}^{T(r)} v(s)^{p^{*}} s^{n-1} d s=\int_{0}^{r} u(s)^{p^{*}} s^{n-1} d s \geq u(r)^{p^{*}} \frac{r^{n}}{n} \quad \text { for } r \geq 0
$$

whence, by 2.9],

$$
T^{\prime}(r) v(T(r))^{p^{*}} \leq a^{p^{*}} M(r) \quad \text { for } r \geq 0 .
$$

Coupling 2.30 and 2.31 implies that

$$
\begin{aligned}
C_{8} \sqrt{\frac{\delta(u)}{\varepsilon^{p^{\sharp} / p}}} & \geq \int_{J}\left|T^{\prime}(r)-M(r)\right| v(T(r))^{p^{*}(1-1 / 2 n)} d r \\
& \geq v\left(T\left(r_{2}\right)\right)^{p^{*}(1-1 / 2 n)} \int_{J}\left|T^{\prime}(r)-M(r)\right| d r .
\end{aligned}
$$

Now, observe that

$$
v\left(T\left(r_{2}\right)\right)^{p^{*}}=\frac{a^{p^{*}}}{\left(1+T\left(r_{2}\right)^{p^{\prime}}\right)^{n}} \geq \frac{a^{p^{*}}}{2^{n}} \min \left\{1, \frac{1}{T\left(r_{2}\right)^{p^{\prime} n}}\right\} .
$$

Formula 2.23 can be used to deduce that

$$
\begin{aligned}
(4 \varepsilon)^{n /(n-p)} & =n \omega_{n} \int_{r_{2}}^{1} u(s)^{p^{*}} s^{n-1} d s=n \omega_{n} \int_{T\left(r_{2}\right)}^{\infty} v(s)^{p^{*}} s^{n-1} d s \\
& =n \omega_{n} a^{p^{*}} \int_{T\left(r_{2}\right)}^{\infty} \frac{s^{n-1}}{\left(1+s^{p^{\prime}}\right)^{n}} d s \\
& \leq n \omega_{n} a^{p^{*}} \int_{T\left(r_{2}\right)}^{\infty} s^{n-1-n p^{\prime}} d s=(p-1) \omega_{n} a^{p^{*}} T\left(r_{2}\right)^{-n /(p-1)},
\end{aligned}
$$

whence

$$
T\left(r_{2}\right) \leq \frac{C_{9}}{\varepsilon^{(p-1) /(n-p)}}
$$

for some constant $C_{9}$. From 2.33, combined with 2.34, we infer that

$$
v\left(T\left(r_{2}\right)\right)^{p^{*}} \geq \frac{a^{p^{*}} \varepsilon^{p^{*}}}{2^{n} C_{9}^{p^{\prime} n}}
$$

provided that $\varepsilon<\varepsilon(p, n)$ for a sufficiently small $\varepsilon(p, n)$. From this inequality and 2.32 one gets

$$
\int_{J}\left|T^{\prime}(r)-M(r)\right| d r \leq C_{10} \sqrt{\frac{\delta(u)}{\varepsilon^{p^{\sharp} / p+(2-1 / n) p^{*}}}} .
$$


As far as $\int_{I}\left|T^{\prime}(r)-M(r)\right| d r$ is concerned, by 2.29) and 2.27) again one has

$$
\begin{aligned}
2 C_{7} n^{\prime} \frac{\delta(u)}{\varepsilon p^{\sharp} / p} & \geq \int_{I} \frac{\left(T^{\prime}(r)-M(r)\right)^{2}}{T^{\prime}(r)^{2} v(T(r))^{p^{*} / n}}\left(\frac{T^{\prime}(r)}{M(r)}\right)^{2-1 / n} v(T(r))^{p^{*} / n} T^{\prime}(r)^{1 / n} M(r)^{1 / n^{\prime}} d r \\
& \geq \frac{1}{C_{6}}\left(\int_{I}\left|T^{\prime}(r)-M(r)\right|\left(\frac{v(T(r))^{p^{*}} T^{\prime}(r)}{M(r)}\right)^{1 / 2 n} d r\right)^{2} \\
& =\frac{1}{C_{6}}\left(\int_{I}\left|T^{\prime}(r)-M(r)\right|\left(\frac{u(r)^{p^{*}} r^{n}}{T(r)^{n}}\right)^{1 / 2 n} d r\right)^{2} .
\end{aligned}
$$

Note that in the last inequality we have made use of 2.9]. Inasmuch as $T(r) \leq T\left(r_{2}\right)$ for $r \in I$, inequalities 2.36, 2.34 and 2.25 ensure that

$$
\varepsilon^{p^{*} / 2 n} \int_{I}\left|T^{\prime}(r)-M(r)\right| d r \leq C_{11} \sqrt{\delta(u) / \varepsilon^{p^{\sharp} / p}}
$$

for some positive constant $C_{11}$. Coupling 2.35) and 2.37) yields

$$
\int_{r_{1}}^{r_{2}}\left|T^{\prime}(r)-M(r)\right| d r \leq C_{12} \sqrt{\delta(u) / \varepsilon^{\omega_{0}}}
$$

for some positive constant $C_{12}$ where

$$
\omega_{0}=\frac{p^{\sharp}}{p}+\left(2-\frac{1}{n}\right) p^{*} .
$$

Step IV. Conclusion. Here, we single out the extremal (2.2) to be used in estimating $\lambda(u)$. Set $k=M\left(r_{2}\right)$ and define $v_{0}=[0, \infty) \rightarrow[0, \infty)$ as

$$
v_{0}(r)=k^{(n-p) / p} v(k r) \quad \text { for } r \geq 0 .
$$

Clearly, $v_{0}$ is an extremal function in the Bliss inequality, still fulfilling $n \omega_{n} \int_{0}^{\infty} v_{0}(r)^{p^{*}} r^{n-1} d r=1$. Consequently, by 2.23 , there exists a constant $C_{13}$ such that

$$
\begin{aligned}
\lambda(u) & \leq n \omega_{n} \int_{0}^{\infty}\left|u(r)-v_{0}(r)\right|^{p^{*}} r^{n-1} d r \\
& \leq C_{13}\left(\varepsilon^{n /(n-p)}+\int_{r_{2}}^{\infty} v_{0}(r)^{p^{*}} r^{n-1} d r+\int_{0}^{r_{1}} v_{0}(r)^{p^{*}} r^{n-1} d r\right. \\
& \left.+\int_{r_{1}}^{r_{2}}\left|u(r)-v_{0}(r)\right|^{p^{*}} r^{n-1} d r\right) .
\end{aligned}
$$

The point is to estimate the last three integrals. As far as the first one is concerned, owing to 2.23 one has

$$
\begin{gathered}
\int_{r_{2}}^{\infty} v_{0}(r)^{p^{*}} r^{n-1} d r=k^{n} \int_{r_{2}}^{\infty} v(k r)^{p^{*}} r^{n-1} d r=\int_{k r_{2}}^{\infty} v(r)^{p^{*}} r^{n-1} d r \\
=\int_{T\left(r_{2}\right)}^{\infty} v(r)^{p^{*}} r^{n-1} d r=\frac{1}{n \omega_{n}}-\int_{0}^{r_{2}} u(r)^{p^{*}} r^{n-1} d r=\frac{(4 \varepsilon)^{n /(n-p)}}{n \omega_{n}} .
\end{gathered}
$$


Next, we have

$$
\int_{0}^{r_{1}} v_{0}(r)^{p^{*}} r^{n-1} d r=\int_{0}^{r_{1} M\left(r_{2}\right)} v(r)^{p^{*}} r^{n-1} d r .
$$

If $M\left(r_{1}\right) \leq M\left(r_{2}\right)$, then

$$
\begin{aligned}
M\left(r_{2}\right) r_{1} & =T\left(r_{1}\right)+r_{1}\left(M\left(r_{2}\right)-M\left(r_{1}\right)\right)=T\left(r_{1}\right)+r_{1} \int_{r_{1}}^{r_{2}} \frac{T^{\prime}(r)-M(r)}{r} d r \\
& \leq T\left(r_{1}\right)+\int_{r_{1}}^{r_{2}}\left|T^{\prime}(r)-M(r)\right| d r \leq T\left(r_{1}\right)+C_{12} \sqrt{\delta(u) / \varepsilon^{\omega_{0}}}
\end{aligned}
$$

where we have exploited 2.38) in the last inequality. Consequently, 2.41, 2.42 and 2.23) tell us that

$$
\begin{aligned}
\int_{0}^{r_{1}} v_{0}(r)^{p^{*}} r^{n-1} d r & \leq(4 \varepsilon)^{p^{*} / p}+a^{p^{*}} \int_{T\left(r_{1}\right)}^{T\left(r_{1}\right)+C_{12} \sqrt{\delta(u) / \varepsilon^{\omega_{0}}}} \frac{r^{n-1}}{\left(1+r^{p^{\prime}}\right)^{n}} d r \\
& \leq C_{14}\left(\varepsilon^{p^{*} / p}+\sqrt{\delta(u) / \varepsilon^{\omega_{0}}}\right)
\end{aligned}
$$

for some positive constant $C_{14}$. Inequality 2.43) continues to hold even if $M\left(r_{2}\right) \leq$ $M\left(r_{1}\right)$, since $M\left(r_{2}\right) r_{1}=T\left(r_{1}\right) M\left(r_{2}\right) / M\left(r_{1}\right)$, and hence, by 2.41) and 2.23,

$$
\begin{aligned}
n \omega_{n} \int_{0}^{r_{1}} v_{0}(r)^{p^{*}} r^{n-1} d r & \leq n \omega_{n} \int_{0}^{T\left(r_{1}\right)} v(r)^{p^{*}} r^{n-1} d r=n \omega_{n} \int_{0}^{r_{1}} u(r)^{p^{*}} r^{n-1} d r \\
& =(4 \varepsilon)^{p^{*} / p}
\end{aligned}
$$

The estimate for the last integral in 2.39 is the most delicate. Thanks to 2.9),

$$
\begin{aligned}
& \int_{r_{1}}^{r_{2}}\left|u(r)-v_{0}(r)\right|^{p^{*}} r^{n-1} d r=\int_{r_{1}}^{r_{2}}\left|u(r)-k^{(n-p) / p} v(k r)\right|^{p^{*}} r^{n-1} d r \\
& =\int_{r_{1}}^{r_{2}}\left|\left(v(T(r))^{p^{*}} M(r)^{n-1} T^{\prime}(r)\right)^{1 / p^{*}}-\left(k^{n} v(k r)^{p^{*}}\right)^{1 / p^{*}}\right|^{p^{*}} r^{n-1} d r \\
& \leq \int_{r_{1}}^{r_{2}}\left|v(T(r))^{p^{*}} M(r)^{n-1} T^{\prime}(r)-k^{n} v(k r)^{p^{*}}\right| r^{n-1} d r \\
& \leq \int_{r_{1}}^{r_{2}} v(T(r))^{p^{*}} T(r)^{n-1}\left|T^{\prime}(r)-M(r)\right| d r+\int_{r_{1}}^{r_{2}} v(T(r))^{p^{*}}\left|M(r)^{n}-k^{n}\right| r^{n-1} d r \\
& \quad+k^{n} \int_{r_{1}}^{r_{2}}\left|v(T(r))^{p^{*}}-v(k r)^{p^{*}}\right| r^{n-1} d r .
\end{aligned}
$$

Since $v(T(r))^{p^{*}} T(r)^{n-1}$ is bounded from above in terms of $p$ and $n$ only, one has, by (2.38),

$$
\int_{r_{1}}^{r_{2}} v(T(r))^{p^{*}} T(r)^{n-1}\left|T^{\prime}(r)-M(r)\right| d r \leq C_{15} \sqrt{\delta(u) / \varepsilon^{\omega_{0}}}
$$


for some constant $C_{15}$. The boundedness of $v(T(r))^{p^{*}} T(r)^{n-1}$ again implies that

$$
\begin{aligned}
\int_{r_{1}}^{r_{2}} v(T(r))^{p^{*}}\left|M(r)^{n}-k^{n}\right| r^{n-1} d r \leq C_{16} \int_{r_{1}}^{r_{2}} \frac{\left|M(r)^{n}-k^{n}\right|}{M(r)^{n-1}} d r \\
\leq n C_{16} \int_{r_{1}}^{r_{2}}\left|M(r)-M\left(r_{2}\right)\right| \frac{\max \left\{M(r)^{n-1}, M\left(r_{2}\right)^{n-1}\right\}}{M(r)^{n-1}} d r
\end{aligned}
$$

for some constant $C_{16}$. By 2.34,

$$
\frac{M\left(r_{2}\right)}{M(r)}=\frac{T\left(r_{2}\right)}{T(r)} \frac{r}{r_{2}} \leq \frac{T\left(r_{2}\right)}{T(r)} \leq \frac{T\left(r_{2}\right)}{T\left(r_{1}\right)} \leq \frac{C_{9}}{\varepsilon^{(p-1) /(n-p)} T\left(r_{1}\right)} \quad \text { for } r \in\left[r_{1}, r_{2}\right],
$$

and, by 2.23$)$,

$$
\frac{(4 \varepsilon)^{p^{*} / p}}{n \omega_{n}}=\int_{0}^{T\left(r_{1}\right)} v(r)^{p^{*}} r^{n-1} d r=a^{p^{*}} \int_{0}^{T\left(r_{1}\right)} \frac{r^{n-1}}{\left(1+r^{p^{\prime}}\right)^{n}} d r \leq \frac{a^{p^{*}}}{n} T\left(r_{1}\right)^{n},
$$

whence

$$
\frac{M\left(r_{2}\right)}{M(r)} \leq \frac{C_{17}}{\varepsilon^{p /(n-p)}} \quad \text { for } r \in\left[r_{1}, r_{2}\right],
$$

for some constant $C_{17}$. Combining 2.46) and 2.47) yields

$$
\int_{r_{1}}^{r_{2}} v(T(r))^{p^{*}}\left|M(r)^{n}-k^{n}\right| r^{n-1} d r \leq \frac{C_{18}}{\varepsilon^{p^{\sharp}}} \int_{r_{1}}^{r_{2}}\left|M(r)-M\left(r_{2}\right)\right| d r
$$

for some constant $C_{18}$. On the other hand,

$$
\begin{aligned}
\int_{r_{1}}^{r_{2}}\left|M(r)-M\left(r_{2}\right)\right| d r \leq \int_{r_{1}}^{r_{2}} d r \int_{r}^{r_{2}} \frac{\left|T^{\prime}(t)-M(t)\right|}{t} d t \\
=\int_{r_{1}}^{r_{2}} \frac{\left|T^{\prime}(t)-M(t)\right|}{t} d t \int_{r_{1}}^{t} d r \leq \int_{r_{1}}^{r_{2}}\left|T^{\prime}(t)-M(t)\right| d t .
\end{aligned}
$$

Thus, thanks to (2.48), 2.49) and (2.38),

$$
\int_{r_{1}}^{r_{2}} v(T(r))^{p^{*}}\left|M(r)^{n}-k^{n}\right| r^{n-1} d r \leq C_{19} \sqrt{\delta(u) / \varepsilon^{\omega_{0}+2 p^{\sharp}}}
$$

for some constant $C_{19}$. Finally, since the function $v^{p^{*}}$ is Lipschitz continuous in $[0, \infty)$ (with Lipschitz constant not exceeding $n p^{\prime} a^{p^{*}}$ ),

$$
\left|v(T(r))^{p^{*}}-v\left(M\left(r_{2}\right) r\right)^{p^{*}}\right| \leq n p^{\prime} a^{p^{*}} r\left|M(r)-M\left(r_{2}\right)\right| \quad \text { for } r \geq 0 .
$$

Hence, via 2.34, 2.49) and 2.38, we get

$$
\begin{aligned}
& \int_{r_{1}}^{r_{2}}\left|v(T(r))^{p^{*}}-v(k r)^{p^{*}}\right| k^{n} r^{n-1} d r \leq n p^{\prime} a^{p^{*}} T\left(r_{2}\right)^{n} \int_{r_{1}}^{r_{2}}\left|M(r)-M\left(r_{2}\right)\right| d r \\
& \quad \leq \frac{n p^{\prime} a^{p^{*}} C_{9}^{n}}{\varepsilon^{n(p-1) /(n-p)}} \int_{r_{1}}^{r_{2}}\left|M(r)-M\left(r_{2}\right)\right| d r \leq \frac{n p^{\prime} a^{p^{*}} C_{9}^{n}}{\varepsilon^{n(p-1) /(n-p)}} \int_{r_{1}}^{r_{2}}\left|T^{\prime}(r)-M(r)\right| d r \\
& \quad \leq C_{20} \sqrt{\delta(u) / \varepsilon^{\omega_{0}+2 p^{\sharp}}}
\end{aligned}
$$


for some constant $C_{20}$. Combining 2.44, 2.45, 2.50) and 2.51) tells us that

$$
\int_{r_{1}}^{r_{2}}\left|u(r)-v_{0}(r)\right|^{p^{*}} r^{n-1} d r \leq C_{21} \sqrt{\delta(u) / \varepsilon^{\omega_{1}}},
$$

where

$$
\omega_{1}=\omega_{0}+2 p^{\sharp} .
$$

From 2.39, 2.40, 2.43 and 2.52 we conclude that

$$
\lambda(u) \leq C_{22}\left\{\varepsilon^{n /(n-p)}+\sqrt{\delta(u) / \varepsilon^{\omega_{1}}}\right\}
$$

for some constant $C_{22}$. The choice

$$
\varepsilon^{\omega_{1}+2 n /(n-p)}=\delta(u),
$$

which is compatible with 2.24) provided that 2.6 holds for a sufficiently small $\varepsilon(p, n)$, yields

$$
\lambda(u) \leq C_{23} \delta(u)^{\omega},
$$

where

$$
\omega=\frac{n}{(n-p) \omega_{1}+2 n}
$$

and $C_{23}$ is a suitable constant. Obviously, inequality 2.53 continues to hold for some constant even if 2.6 is violated, if $\delta(u) \geq \varepsilon(p, n)$. Actually, since $\lambda(u) \leq 2^{p^{*}}$,

$$
\delta(u) \geq \varepsilon(p, n) \geq \varepsilon(p, n) 2^{-p^{*} / \omega} \lambda(u)^{1 / \omega} .
$$

This proves 2.5 with

$$
\beta=\frac{1}{\omega}=3+4 p-\frac{3 p+1}{n} .
$$

\section{The case of $n$-symmetric functions}

As recalled in the Introduction, the Pólya-Szegö inequality 1.6 does not enjoy the stability property which would immediately imply Theorem 1 via the one-dimensional Theorem 2 and from inequality (1.8). Indeed, although equality trivially holds in 1.6 whenever $f$ is spherically symmetric, the sole gap between $\|\nabla f\|_{L^{p}\left(\mathbb{R}^{n}\right)}$ and $\left\|\nabla f^{\star}\right\|_{L^{p}\left(\mathbb{R}^{n}\right)}$ is not sufficient to estimate the asymmetry of $f$ measured as a distance (in some integral norm) of $f$ from a (translate of) $f^{\star}$. Such a distance, in any $L^{q}$ norm with $1 \leq q<p^{*}$, can actually be estimated if information on the measure of the sets $\left\{\left|\nabla f^{\star}\right|<\varepsilon\right\}$ is also retained, as recently shown in [CEFT]. In fact, this result could be used to prove a weaker form of Theorem 1 for functions supported in sets of finite measure and with $p^{*}$ in definition (1.3) replaced by any smaller exponent. The full version of Theorem 1 requires, instead, the quantitative Pólya-Szegö principle for $n$-symmetric functions contained in Theorem 3 below. We say that a function $f: \mathbb{R}^{n} \rightarrow \mathbb{R}$ is $k$-symmetric, with $1 \leq k \leq n$, if there exist $k$ mutually orthogonal hyperplanes such that $f$ is symmetric with respect to 
each of them. Moreover, if $f: \mathbb{R}^{n} \rightarrow[0, \infty)$ is any measurable function satisfying

$$
|\{x: f(x)>t\}|<\infty \quad \text { for } t>0,
$$

its spherically symmetric rearrangement $f^{\star}: \mathbb{R}^{n} \rightarrow[0, \infty)$ is given by

$$
f^{\star}(x)=\sup \left\{t \geq 0:|\{f>t\}|>\omega_{n}|x|^{n}\right\} \quad \text { for } x \in \mathbb{R}^{n} .
$$

Theorem 3. Let $n \geq 2$ and let $1<p<n$. Set $q=\max \{p, 2\}$. Then there exists $a$ positive constant $C$ such that

$$
\int_{\mathbb{R}^{n}}\left|f-f^{\star}\right|^{p^{*}} \leq C\left(\int_{\mathbb{R}^{n}}|f|^{p^{*}}\right)^{p / n}\left(\int_{\mathbb{R}^{n}}\left|\nabla f^{\star}\right|^{p}\right)^{1 / q^{\prime}}\left(\int_{\mathbb{R}^{n}}|\nabla f|^{p}-\int_{\mathbb{R}^{n}}\left|\nabla f^{\star}\right|^{p}\right)^{1 / q}
$$

for every nonnegative $f \in W^{1, p}\left(\mathbb{R}^{n}\right)$ which is symmetric with respect to the coordinate hyperplanes.

It is clear that, up to a rigid motion, the inequality of Theorem 3 holds for any $n$-symmetric function.

Thanks to inequalities (1.7) and (1.8), a combination of Theorems 2 and 3 easily yields inequality (1.4) for $n$-symmetric functions.

Corollary 4. Let $n \geq 2$ and let $1<p<n$. Then there exists a constant $\kappa>0$ such that (1.4) holds for every nonnegative $n$-symmetric function $f \in W^{1, p}\left(\mathbb{R}^{n}\right)$, with $\alpha=\beta$, where $\beta$ is as in (2.54).

In analogy with 2.4 (and again with a slight abuse of notation), we define

$$
\delta(f)=\frac{\|\nabla f\|_{L^{p}\left(\mathbb{R}^{n}\right)}}{S(p, n)\|f\|_{L^{p^{*}}\left(\mathbb{R}^{n}\right)}}-1
$$

for $f \in W^{1, p}\left(\mathbb{R}^{n}\right)$. Inequality 1.4 can then be written as

$$
\lambda(f) \leq C \delta(f)^{1 / \alpha}
$$

where $C=(1 / \kappa)^{1 / \alpha}$.

Proof of Corollary 4 We may assume, without loss of generality, that $\|f\|_{L^{p^{*}}\left(\mathbb{R}^{n}\right)}=1$ and $f$ is symmetric with respect to the coordinate hyperplanes; in fact, both $\delta(f)$ and $\lambda(f)$ are invariant under rescaling, multiplication by a constant and rigid motions. Suppose, for the time being, that $\delta(f) \leq 1 / S(p, n)$. Then

$$
S(p, n) \leq\left\|\nabla f^{\star}\right\|_{L^{p}\left(\mathbb{R}^{n}\right)} \leq\|\nabla f\|_{L^{p}\left(\mathbb{R}^{n}\right)} \leq 1+S(p, n) .
$$

We have

$$
\begin{aligned}
\lambda(f)^{1 / p^{*}} \leq \lambda\left(f^{\star}\right)^{1 / p^{*}}+\left\|f-f^{\star}\right\|_{L^{p^{*}}\left(\mathbb{R}^{n}\right)} & \leq C\left(\left(\left\|\nabla f^{\star}\right\|_{L^{p^{*}}\left(\mathbb{R}^{n}\right)}-S(p, n)\right)^{1 / \beta p^{*}}\right. \\
& \left.\quad+\left\|\nabla f^{\star}\right\|_{L^{p}\left(\mathbb{R}^{n}\right)}^{p / q^{\prime} p^{*}}\left(\|\nabla f\|_{L^{p}\left(\mathbb{R}^{n}\right)}^{p}-\left\|\nabla f^{\star}\right\|_{L^{p}\left(\mathbb{R}^{n}\right)}^{p}\right)^{1 / q p^{*}}\right)
\end{aligned}
$$


for some constant $C$, where the first inequality is just a consequence of the triangle inequality, and the second one follows from Theorems 2 and 3 . Inequalities 3.4 ensure that

$$
\|\nabla f\|_{L^{p}\left(\mathbb{R}^{n}\right)}^{p}-\left\|\nabla f^{\star}\right\|_{L^{p}\left(\mathbb{R}^{n}\right)}^{p} \leq C\left(\|\nabla f\|_{L^{p}\left(\mathbb{R}^{n}\right)}-\left\|\nabla f^{\star}\right\|_{L^{p}\left(\mathbb{R}^{n}\right)}\right)
$$

for some constant $C$. Combining (3.5), 3.6, 1.7) and (1.8) yields

$$
\lambda(f)^{1 / p^{*}} \leq C\left(\delta(f)^{1 / \beta p^{*}}+\delta(f)^{1 / q p^{*}}\right)
$$

for some constant $C$. Hence, inequality (1.4) follows with $\alpha=\beta$, since $\beta>q$ by (2.54). If $\delta(f)>1 / S(p, n)$, the assertion is a straightforward consequence of the inequality $\lambda(f) \leq 2^{p^{*}}$.

The following estimate for the distance between functions in $L^{q}\left(\mathbb{R}^{n}\right)$ involving the measure of the symmetric difference of their level sets will be exploited in the proof of Theorem 3 .

Lemma 5. Let $q \geq 1$. Given any nonnegative functions $f, g \in L^{q}\left(\mathbb{R}^{n}\right)$, set

$$
E_{t}=\{f>t\} \triangle\{g>t\},
$$

where $\triangle$ stands for the symmetric difference of sets. Then

$$
\int_{\mathbb{R}^{n}}|f-g|^{q} \leq q \int_{0}^{\infty}\left|E_{t}\right| t^{q-1} d t .
$$

Proof. The layer-cake formula and Fubini's theorem yield

$$
\begin{aligned}
\int_{\mathbb{R}^{n}}|f-g|^{q} & =\int_{\mathbb{R}^{n}}|f(x)-g(x)|^{q-1}\left|\int_{0}^{\infty} \chi_{\{f>t\}}(x) d t-\int_{0}^{\infty} \chi_{\{g>t\}}(x) d t\right| d x \\
& \leq \int_{\mathbb{R}^{n}}|f(x)-g(x)|^{q-1} \int_{0}^{\infty}\left|\chi_{\{f>t\}}(x)-\chi_{\{g>t\}}(x)\right| d t d x \\
& =\int_{0}^{\infty} \int_{E_{t}}|f(x)-g(x)|^{q-1} d x d t .
\end{aligned}
$$

Here $\chi_{G}$ denotes the characteristic function of the set $G$. Thus, since

$$
\begin{aligned}
\int_{E_{t}}|f-g|^{q-1} & =\int_{\{g \leq t<f\}}|f-g|^{q-1}+\int_{\{f \leq t<g\}}|f-g|^{q-1} \\
& \leq \int_{\{g \leq t<f\}} f^{q-1}+\int_{\{f \leq t<g\}} g^{q-1}
\end{aligned}
$$

for any $t \geq 0$, one has

$$
\int_{\mathbb{R}^{n}}|f-g|^{q} \leq \int_{0}^{\infty} \int_{\{g \leq t<f\}} f(x)^{q-1} d x d t+\int_{0}^{\infty} \int_{\{f \leq t<g\}} g(x)^{q-1} d x d t .
$$


Now,

$$
\begin{aligned}
\int_{0}^{\infty} \int_{\{g \leq t<f\}} f(x)^{q-1} d x d t & =\int_{0}^{\infty} \int_{\mathbb{R}^{n}} \chi_{\{g \leq t<f\}}(x) \int_{0}^{\infty} \chi_{\left[0, f(x)^{q-1}\right]}(s) d s d x d t \\
& =\int_{0}^{\infty} \int_{\mathbb{R}^{n}} \int_{0}^{\infty} \chi_{\left\{f>\max \left\{t, s^{1 /(q-1)}\right\} ; t \geq g\right\}}(x) d s d x d t .
\end{aligned}
$$

Another application of Fubini's theorem ensures that

$$
\begin{aligned}
& \int_{0}^{\infty} \int_{\mathbb{R}^{n}} \int_{0}^{\infty} \chi_{\left\{f>\max \left\{t, s^{1 /(q-1)}\right\} ; t \geq g\right\}}(x) d s d x d t \\
& =\int_{0}^{\infty} \int_{\mathbb{R}^{n}} \int_{0}^{t^{q-1}} \chi_{\{g \leq t<f\}}(x) d s d x d t+\int_{\mathbb{R}^{n}} \int_{0}^{\infty} \int_{t^{q-1}}^{\infty} \chi_{\left\{f>s^{1 /(q-1)} ; t \geq g\right\}}(x) d s d t d x \\
& =\int_{0}^{\infty} t^{q-1}|\{g \leq t<f\}| d t+\int_{\mathbb{R}^{n}} \int_{0}^{\infty} \int_{0}^{s^{1 /(q-1)}} \chi_{\left\{f>s^{1 /(q-1)} ; t \geq g\right\}}(x) d t d s d x \\
& \leq \int_{0}^{\infty} t^{q-1}|\{g \leq t<f\}| d t+\int_{\mathbb{R}^{n}} \int_{0}^{\infty} \int_{0}^{s^{1 /(q-1)}} \chi_{\left\{g \leq s^{1 /(q-1)}<f\right\}}(x) d t d s d x \\
& =\int_{0}^{\infty} t^{q-1}|\{g \leq t<f\}| d t+\int_{0}^{\infty} s^{1 /(q-1)}\left|\left\{g \leq s^{1 /(q-1)}<f\right\}\right| d s .
\end{aligned}
$$

The change of variable $s=\tau^{q-1}$ in the last integral yields

$$
\int_{0}^{\infty} s^{1 /(q-1)}\left|\left\{g \leq s^{1 /(q-1)}<f\right\}\right| d s=(q-1) \int_{0}^{\infty} \tau^{q-1}|\{g \leq \tau<f\}| d \tau .
$$

Thus, combining (3.9) and 3.10) entails that

$$
\int_{0}^{\infty} \int_{\{g \leq t<f\}} f(x)^{q-1} d x d t \leq q \int_{0}^{\infty} t^{q-1}|\{g \leq t<f\}| d t .
$$

Since $\left|E_{t}\right|=|\{g \leq t<f\}|+|\{f \leq t<g\}|$ for $t \geq 0$, inequality (3.7) follows from (3.8), from (3.11) and from an analogous estimate for the second integral on the right-hand side of 3.8 .

Proof of Theorem 3 Assume, without loss of generality, that $\|f\|_{L^{p^{*}}\left(\mathbb{R}^{n}\right)}=1$. By the coarea formula,

$$
\mu(t)=|\{f>t\} \cap\{\nabla f=0\}|+\int_{t}^{\infty} \int_{\{f=s\}} \frac{d \mathscr{H}^{n-1}}{|\nabla f|} d s \quad \text { for } t>0,
$$

where $\mathscr{H}^{n-1}$ denotes the (n-1)-dimensional Hausdorff measure (see e.g. [BZ] or [CF1]). Hence,

$$
-\mu^{\prime}(t) \geq \int_{\{f=t\}} \frac{d \mathscr{H}^{n-1}}{|\nabla f|} \quad \text { for a.e. } t>0 .
$$


One has

$$
\mathscr{H}^{n-1}(\{f=t\})=P(\{f>t\}) \quad \text { for a.e. } t>0,
$$

where $P$ stands for perimeter in the sense of geometric measure theory (see e.g. [BZ] equation (19)]). Then an application of the coarea formula again, Hölder's inequality and 3.12 entail that

$$
\begin{aligned}
\int_{\mathbb{R}^{n}}|\nabla f|^{p} & =\int_{0}^{\infty} \int_{\{f=t\}}|\nabla f|^{p-1} d \mathscr{H}^{n-1} d t \geq \int_{0}^{\infty} \frac{\mathscr{H}^{n-1}(\{f=t\})^{p}}{\left(\int_{\{f=t\}} \frac{d \mathscr{H}^{n-1}}{|\nabla f|}\right)^{p-1}} d t \\
& \geq \int_{0}^{\infty} \frac{\mathscr{H}^{n-1}(\{f=t\})^{p}}{\left(-\mu^{\prime}(t)\right)^{p-1}} d t=\int_{0}^{\infty} \frac{P(\{f>t\})^{p}}{\left(-\mu^{\prime}(t)\right)^{p-1}} d t .
\end{aligned}
$$

The quantitative isoperimetric inequality of [FMP1] tells us that there exists a constant $\kappa_{0}$, depending only on $n$, such that

$$
n \omega_{n}|E|^{1 / n^{\prime}}\left[1+\kappa_{0}\left(\inf \left\{\frac{|E \triangle B|}{|E|}: B \text { ball, }|B|=|E|\right\}\right)^{2}\right] \leq P(E)
$$

for every measurable subset of $\mathbb{R}^{n}$ having finite measure and perimeter. If, in addition, $E$ is symmetric about $n$ orthogonal hyperplanes containing 0 , then

$$
\inf \left\{\frac{|E \Delta B|}{|E|}: B \text { ball, }|B|=|E|\right\} \geq \frac{1}{2^{n}} \frac{\left|E \Delta E^{\star}\right|}{|E|},
$$

where $E^{\star}$ denotes the ball, centered at 0 , such that $\left|E^{\star}\right|=|E|$ (see [FMP1] Lemma 2.2]). Since $f$ is $n$-symmetric, so are its level sets $\{f>t\}$ for $t>0$; moreover, the ball $\{f>t\}^{\star}$ agrees with $\left\{f^{\star}>t\right\}$ for every $t>0$. Consequently, from 3.13 and from 3.14 and 3.15 applied with $E=\{f>t\}$, we deduce that

$$
\int_{\mathbb{R}^{n}}|\nabla f|^{p} \geq\left(n \omega_{n}^{1 / n}\right)^{p} \int_{0}^{\infty} \frac{\mu(t)^{p / n^{\prime}}}{\left(-\mu^{\prime}(t)\right)^{p-1}}\left(1+\frac{\kappa_{0}}{4^{n}}\left(\frac{\left|F_{t}\right|}{\mu(t)}\right)^{2}\right)^{p} d t,
$$

where we have set $F_{t}=\{f>t\} \triangle\left\{f^{\star}>t\right\}$ for $t>0$. Now, note that when 3.13 is applied with $f$ replaced by $f^{\star}$, equality holds in the first equality because $\left|\nabla f^{\star}\right|$ is constant on $\left\{f^{\star}=t\right\}$ for a.e. $t>0$, and equality holds in the second inequality since also (3.12) turns into an equality in this case (see e.g. [CF1, Lemma 3.2]). Thus, inasmuch as $P\left(\left\{f^{\star}>t\right\}\right)=n \omega_{n}^{1 / n} \mu(t)^{1 / n^{\prime}}$ for a.e. $t>0$, one has

$$
\int_{\mathbb{R}^{n}}\left|\nabla f^{\star}\right|^{p}=\left(n \omega_{n}^{1 / n}\right)^{p} \int_{0}^{\infty} \frac{\mu(t)^{p / n^{\prime}}}{\left(-\mu^{\prime}(t)\right)^{p-1}} d t .
$$

Since $(1+s)^{p} \geq 1+p s$ for $s \geq 0$, we infer from (3.16) and 3.17) that

$$
\int_{\mathbb{R}^{n}}|\nabla f|^{p}-\int_{\mathbb{R}^{n}}\left|\nabla f^{\star}\right|^{p} \geq \kappa \int_{0}^{\infty}\left(\frac{\left|F_{t}\right|}{\mu(t)}\right)^{2} \frac{\mu(t)^{p / n^{\prime}}}{\left(-\mu^{\prime}(t)\right)^{p-1}} d t
$$


for some positive constant $\kappa$. By Lemma 5

$$
\int_{\mathbb{R}^{n}}\left|f-f^{\star}\right|^{p^{*}} \leq p^{*} \int_{0}^{\infty}\left|F_{t}\right| t^{p^{*}-1} d t
$$

The point is thus to estimate the right-hand side of 3.19) in terms of the right-hand side of 3.18. We have

$$
1=\int_{\mathbb{R}^{n}} f^{p^{*}} \geq \int_{\{f>t\}} f^{p^{*}} \geq t^{p^{*}} \mu(t) \quad \text { for } t>0,
$$

whence $\mu(t)^{p / n} t^{p^{*}-p} \leq 1$ for every $t>0$. Thus, by 3.19) and by Hölder's inequality,

$$
\begin{aligned}
\int_{\mathbb{R}^{n}}\left|f-f^{\star}\right|^{p^{*}} \leq p^{*} \int_{0}^{\infty} \frac{\left|F_{t}\right|}{\mu(t)} \mu(t)^{1-p / n} t^{p-1} d t \\
\quad \leq p^{*}\left(\int_{0}^{\infty}\left(\frac{\left|F_{t}\right|}{\mu(t)}\right)^{p} \frac{\mu(t)^{p / n^{\prime}}}{\left(-\mu^{\prime}(t)\right)^{p-1}} d t\right)^{1 / p}\left(\int_{0}^{\infty} \frac{-\mu^{\prime}(t)}{\mu(t)^{p / n}} t^{p} d t\right)^{1 / p^{\prime}}
\end{aligned}
$$

We claim that there exists a constant $C$ such that

$$
\int_{0}^{\infty} \frac{-\mu^{\prime}(t)}{\mu(t)^{p / n}} t^{p} d t \leq C \int_{\mathbb{R}^{n}}\left|\nabla f^{\star}\right|^{p}
$$

To verify 3.21), fix any $\vartheta \in\left(1 / p^{\prime}, 1 / n^{\prime}\right)$. Then

$$
\begin{aligned}
t^{p} & =\left(\int_{0}^{t} d s\right)^{p} \leq\left(\int_{0}^{t} \frac{\left(-\mu^{\prime}(s)\right)}{\mu(s)^{\vartheta p^{\prime}}} d s\right)^{p-1}\left(\int_{0}^{t} \frac{\mu(s)^{\vartheta p}}{\left(-\mu^{\prime}(s)\right)^{p-1}} d s\right) \\
& \leq \frac{1}{\left(\left(\vartheta p^{\prime}-1\right) \mu(t)^{\vartheta p^{\prime}-1}\right)^{p-1}} \int_{0}^{t} \frac{\mu(s)^{\vartheta p}}{\left(-\mu^{\prime}(s)\right)^{p-1}} d s,
\end{aligned}
$$

by Hölder's inequality. Therefore,

$$
\begin{aligned}
\int_{0}^{\infty} \frac{\left(-\mu^{\prime}(t)\right) t^{p}}{\mu(t)^{p / n}} d t & \leq \frac{1}{\left(\vartheta p^{\prime}-1\right)^{p-1}} \int_{0}^{\infty} \frac{\left(-\mu^{\prime}(t)\right)}{\mu(t)^{\vartheta p+1-p / n^{\prime}}}\left(\int_{0}^{t} \frac{\mu(s)^{\vartheta p}}{\left(-\mu^{\prime}(s)\right)^{p-1}} d s\right) d t \\
& =\frac{1}{\left(\vartheta p^{\prime}-1\right)^{p-1}} \int_{0}^{\infty} \frac{\mu(s)^{\vartheta p}}{\left(-\mu^{\prime}(s)\right)^{p-1}}\left(\int_{s}^{\infty} \frac{\left(-\mu^{\prime}(t)\right)}{\mu(t)^{\vartheta p+1-p / n^{\prime}}} d t\right) d s \\
& \leq \frac{1}{\left(\vartheta p^{\prime}-1\right)^{p-1}\left(p / n^{\prime}-\vartheta p\right)} \int_{0}^{\infty} \frac{\mu(s)^{p / n^{\prime}}}{\left(-\mu^{\prime}(s)\right)^{p-1}} d s
\end{aligned}
$$

Inequality 3.21 follows from 3.22 and 3.17. Combining 3.20 and 3.21 yields

$$
\int_{\mathbb{R}^{n}}\left|f-f^{\star}\right|^{p^{*}} \leq C\left\|\nabla f^{\star}\right\|_{L^{p}\left(\mathbb{R}^{n}\right)}^{p-1}\left(\int_{0}^{\infty}\left(\frac{\left|F_{t}\right|}{\mu(t)}\right)^{p} \frac{\mu(t)^{p / n^{\prime}}}{\left(-\mu^{\prime}(t)\right)^{p-1}} d t\right)^{1 / p} .
$$


When $1<p<2$, by Hölder's inequality,

$$
\begin{aligned}
\int_{0}^{\infty} & \left(\frac{\left|F_{t}\right|}{\mu(t)}\right)^{p} \frac{\mu(t)^{p / n^{\prime}}}{\left(-\mu^{\prime}(t)\right)^{p-1}} d t \\
& \leq\left(\int_{0}^{\infty}\left(\frac{\left|F_{t}\right|}{\mu(t)}\right)^{2} \frac{\mu(t)^{p / n^{\prime}}}{\left(-\mu^{\prime}(t)\right)^{p-1}} d t\right)^{p / 2}\left(\int_{0}^{\infty} \frac{\mu(t)^{p / n^{\prime}}}{\left(-\mu^{\prime}(t)\right)^{p-1}} d t\right)^{1-p / 2},
\end{aligned}
$$

and 3.3 follows via (3.23), 3.24), 3.17) and (3.18). If, instead, $p \geq 2$, then

$$
\left(\frac{\left|F_{t}\right|}{\mu(t)}\right)^{p} \leq 2^{p-2}\left(\frac{\left|F_{t}\right|}{\mu(t)}\right)^{2} \quad \text { for } t>0,
$$

and 3.3 follows from 3.23 , 3.25 and 3.18.

\section{Proof of Theorem 1}

The task of the present section is to accomplish the symmetrization process which we alluded to in Section 1, showing that the proof of inequality (1.4) can always be reduced to the special case of $n$-symmetric functions dealt with in Corollary 4 . This is the content of the following result.

Theorem 6. Let $n \geq 2$ and let $1<p<n$. Then there exists a positive constant $C$ such that for every nonnegative function $f \in W^{1, p}\left(\mathbb{R}^{n}\right)$ there exists a nonnegative $n$-symmetric function $\widehat{f}$ such that

$$
\lambda(f) \leq C \lambda(\widehat{f}), \quad \delta(\widehat{f}) \leq C \delta(f)^{1 / \beta p^{*}},
$$

where $\beta$ is given by 2.54).

Once Theorem 6 is established, Theorem 1 quite easily follows from Corollary 4

Proof of Theorem 1 . Consider first the case where $f$ is nonnegative. Then, by Theorem 6 . there exists an $n$-symmetric function $\widehat{f}$ satisfying (4.1). Inequality (1.4) holds with $f$ replaced by $\widehat{f}$, by Corollary 4 . Owing to (4.1), inequality (1.4) continues to hold with $\alpha=\beta^{2} p^{*}$ for $f$ as well.

Let us now remove the sign assumption on $f$. Consider any function $f \in W^{1, p}\left(\mathbb{R}^{n}\right)$, which, without loss of generality, can be assumed to satisfy $\|f\|_{L^{p^{*}}\left(\mathbb{R}^{n}\right)}=1$ and $\delta(f)$ $\leq 1$. We claim that there exists a constant $C$ such that

$$
\min \left\{\int_{\{f<0\}}|f|^{p^{*}}, \int_{\{f>0\}}|f|^{p^{*}}\right\} \leq C \delta(f) .
$$

Actually, the Sobolev inequality (1.1) applied to $\max \{f, 0\}$ and $\min \{f, 0\}$ yields

$$
S(p, n)^{p}\left(\int_{\{f \gtrless 0\}}|f|^{p^{*}}\right)^{p / p^{*}} \leq \int_{\{f \gtrless 0\}}|\nabla f|^{p},
$$


whence

$$
\left(\int_{\{f>0\}}|f|^{p^{*}}\right)^{p / p^{*}}+\left(\int_{\{f<0\}}|f|^{p^{*}}\right)^{p / p^{*}} \leq \frac{1}{S(p, n)^{p}} \int_{\mathbb{R}^{n}}|\nabla f|^{p}=(1+\delta(f))^{p} .
$$

Since the function $s \mapsto\left(s^{p / p^{*}}+(1-s)^{p / p^{*}}\right)^{1 / p}-1$ is concave in $[0,1]$, there exists a constant $\kappa$ such that

$$
\left(s^{p / p^{*}}+(1-s)^{p / p^{*}}\right)^{1 / p}-1 \geq \kappa \min \{s, 1-s\} .
$$

Thus, inasmuch as $\int_{\{f<0\}}|f|^{p^{*}}=1-\int_{\{f>0\}}|f|^{p^{*}}$, one can infer from (4.3) that

$$
\begin{aligned}
\delta(f) & \geq\left[\left(\int_{\{f>0\}}|f|^{p^{*}}\right)^{p / p^{*}}+\left(\int_{\{f<0\}}|f|^{p^{*}}\right)^{p / p^{*}}\right]^{1 / p}-1 \\
& \geq \kappa \min \left\{\int_{\{f<0\}}|f|^{p^{*}}, \int_{\{f>0\}}|f|^{p^{*}}\right\},
\end{aligned}
$$

that is, 4.2. Now, to fix ideas, assume that the minimum in 4.2$\}$ is $\int_{\{f<0\}}|f|^{p^{*}}$, the other case being completely analogous. By applying (1.4) to $|f|$ and observing that $\delta(f)=$ $\delta(|f|)$, from 4.2 we have

$$
\lambda(f) \leq 2^{p^{*}-1}\left(\lambda(|f|)+\int_{\mathbb{R}^{n}}|f-| f||^{p^{*}}\right) \leq C\left(\delta(f)^{1 / \alpha}+\delta(f)\right) \leq C \delta(f)^{1 / \alpha}
$$

for a suitable constant $C$. Hence, the result easily follows.

The remaining part of the paper is devoted to the proof of Theorem 6 . The argument relies upon some delicate constructions, and is split into separate lemmas. We begin by showing that, when dealing with functions $f$ which are symmetric about orthogonal hyperplanes intersecting in some lower dimensional affine space $S$, the quantity $\lambda(f)$ can be essentially replaced by the expression

$$
\begin{aligned}
& \lambda(f \mid S) \\
& \quad=\inf \left\{\frac{\left\|f-g_{a, b, x_{0}}\right\|_{L^{p^{*}\left(\mathbb{R}^{n}\right)}}^{p^{*}}}{\|f\|_{L^{p^{*}}\left(\mathbb{R}^{n}\right)}^{p^{*}}}:\left\|g_{a, b, x_{0}}\right\|_{L^{p^{*}}\left(\mathbb{R}^{n}\right)}=\|f\|_{L^{p^{*}\left(\mathbb{R}^{n}\right)}}, a \in \mathbb{R}, b>0, x_{0} \in S\right\} .
\end{aligned}
$$

Lemma 7. Let $n \geq 2,1<p<n$ and $f$ be a nonnegative function from $W^{1, p}\left(\mathbb{R}^{n}\right)$. Assume that $f$ is $k$-symmetric, and let $S$ be the intersection of the $k$ hyperplanes of symmetry. Then

$$
\lambda(f \mid S) \leq 3^{p^{*}} \lambda(f)
$$

The proof of Lemma 7 in turn relies upon the technical results contained in Lemmas 8 below. 
Lemma 8. Let $\varphi: \mathbb{R} \rightarrow[0, \infty)$ be increasing on $(-\infty, 0)$ and decreasing on $(0, \infty)$. Define $\Phi:[0, \infty) \rightarrow[0, \infty]$ as

$$
\Phi(h)=\int_{\mathbb{R}} A(|\varphi(t)-\varphi(t-h)|) d t \quad \text { for } h \geq 0,
$$

where $A:[0, \infty) \rightarrow[0, \infty)$ is a l.s.c. increasing function. Then $\Phi$ is decreasing.

Proof. First of all, we may assume that $A$ is continuous, since any 1.s.c. increasing function can be approximated pointwise by an increasing sequence of continuous increasing functions. Then, since $|\min \{t, M\}-\min \{s, M\}| \leq|t-s|$ for any $M>0$ and $s, t \in \mathbb{R}$, we may reduce to the case when $\varphi$ is bounded. A simple approximation argument then shows that we may also assume that there exist $l_{1}<0<l_{2}$ such that $\varphi$ is constant both in $\left(-\infty, l_{1}\right]$ and in $\left[l_{2}, \infty\right)$, and that $\varphi$ is continuous. The function $\Phi$ is trivially affine and increasing in $\left[l_{2}-l_{1}, \infty\right)$, thus we may focus on the interval $\left[0, l_{2}-l_{1}\right]$. For every $h_{1} \in\left(0, l_{2}-l_{1}\right)$, there exists $t_{1} \in\left(0, h_{1}\right)$ satisfying $\varphi\left(t_{1}\right)=\varphi\left(t_{1}-h_{1}\right)$; moreover, $\varphi(t) \geq \varphi\left(t-h_{1}\right)$ if $t<t_{1}$ and $\varphi(t) \leq \varphi\left(t-h_{1}\right)$ if $t>t_{1}$. Let $h_{2} \in\left(h_{1}, l_{2}-l_{1}\right]$. On the one hand,

$$
\begin{aligned}
\int_{-\infty}^{t_{1}} A\left(\left|\varphi(t)-\varphi\left(t-h_{1}\right)\right|\right) d t & =\int_{-\infty}^{t_{1}} A\left(\varphi(t)-\varphi\left(t-h_{1}\right)\right) d t \\
& \leq \int_{-\infty}^{t_{1}} A\left(\left|\varphi(t)-\varphi\left(t-h_{2}\right)\right|\right) d t
\end{aligned}
$$

since $\varphi\left(t-h_{2}\right) \leq \varphi\left(t-h_{1}\right) \leq \varphi(t)$ whenever $t \leq t_{1}$. On the other hand,

$$
\begin{aligned}
\int_{t_{1}}^{\infty} A\left(\left|\varphi(t)-\varphi\left(t-h_{1}\right)\right|\right) d t & =\int_{t_{1}}^{\infty} A\left(\varphi\left(t-h_{1}\right)-\varphi(t)\right) d t \\
& =\int_{t_{1}+h_{2}-h_{1}}^{\infty} A\left(\varphi\left(s-h_{2}\right)-\varphi\left(s+h_{1}-h_{2}\right)\right) d s \\
& \leq \int_{t_{1}+h_{2}-h_{1}}^{\infty} A\left(\left|\varphi\left(s-h_{2}\right)-\varphi(s)\right|\right) d s
\end{aligned}
$$

Thus,

$$
\begin{aligned}
\int_{\mathbb{R}} A\left(\left|\varphi(t)-\varphi\left(t-h_{1}\right)\right|\right) d t & \leq \int_{\left(-\infty, t_{1}\right) \cup\left(t_{1}+h_{2}-h_{1}, \infty\right)} A\left(\left|\varphi(t)-\varphi\left(t-h_{2}\right)\right|\right) d t \\
& \leq \int_{\mathbb{R}} A\left(\left|\varphi(t)-\varphi\left(t-h_{2}\right)\right|\right) d t
\end{aligned}
$$

and the conclusion follows.

Lemma 9. Let $f: \mathbb{R}^{n} \rightarrow[0, \infty)$ be any spherically symmetric function. Given any $y \in \mathbb{R}^{n}$, define $f_{y}: \mathbb{R}^{n} \rightarrow[0, \infty)$ as

$$
f_{y}(x)=f(x-y) \quad \text { for } x \in \mathbb{R}^{n} .
$$


If $A$ is as in Lemma 8 , then

$$
\int_{\mathbb{R}^{n}} A\left(\left|f_{y}(x)-f_{w}(x)\right|\right) d x \leq \int_{\mathbb{R}^{n}} A\left(\left|f_{y}(x)-f_{z}(x)\right|\right) d x
$$

for every $y, z \in \mathbb{R}^{n}$ and for every $w$ lying on the segment joining $y$ and $z$.

Proof. Without loss of generality, we may assume $y=0$ in (4.4); then set $v=z /|z|$, whence $z=|z| v$ and $w=|w| v$. Denote by $H$ the hyperplane orthogonal to $v$ and containing 0 . Then

$$
\begin{gathered}
\int_{\mathbb{R}^{n}} A\left(\left|f(x)-f_{w}(x)\right|\right) d x=\int_{H} \int_{\mathbb{R}} A(|f(x+t v)-f(x-w+t v)|) d t d \mathscr{H}^{n-1}(x) \\
=\int_{H} \int_{\mathbb{R}} A(|f(x+t v)-f(x+(t-|w|) v)|) d t d \mathscr{H}^{n-1}(x) .
\end{gathered}
$$

Fix any $x \in H$, and define $\varphi: \mathbb{R} \rightarrow[0, \infty)$ by

$$
\varphi(t)=f(x+t v) \quad \text { for } t \in \mathbb{R} .
$$

Clearly, the function $\varphi$ satisfies the assumptions of Lemma 8 Hence,

$$
\int_{\mathbb{R}} A\left(\left|\varphi(t)-\varphi\left(t-h_{1}\right)\right|\right) d t \leq \int_{\mathbb{R}} A\left(\left|\varphi(t)-\varphi\left(t-h_{2}\right)\right|\right) d t \quad \text { if } 0<h_{1} \leq h_{2} .
$$

On applying 4.6] with $h_{1}=|w|$ and $h_{2}=|z|$ we get

$\int_{\mathbb{R}} A(|f(x+t v)-f(x+(t-|w|) v)|) d t \leq \int_{\mathbb{R}} A(|f(x+t v)-f(x+(t-|z|) v)|) d t$.

Combining this inequality with (4.5) yields the conclusion.

We now want to prove that, when $f$ is positive, the infima defining $\lambda(f)$ and $\lambda(f \mid S)$ are attained; this proof closely resembles the proof of Lemma B.1 in [FMP2], and we will obtain it in two steps.

Lemma 10. Let $1<p<n$ and let $f$ be any nonnegative function from $L^{p^{*}}\left(\mathbb{R}^{n}\right)$. Then $\lambda(f)$ is a minimum. The same holds true for $\lambda(f \mid S)$ with any affine subspace $S$ of $\mathbb{R}^{n}$.

Proof. We only give the proof for $\lambda(f)$, the other case being analogous; we also assume without loss of generality that $\|f\|_{L^{p^{*}}\left(\mathbb{R}^{n}\right)}=1$.

The proof is in two steps; notice that the sign assumption on $f$ plays a role only in Step II.

Step I. If $\lambda(f)<2$, then $\lambda(f)$ is a minimum. Let us consider a minimizing sequence for $\lambda(f)$, given by the functions

$$
g_{h}(x)=\frac{a_{h}}{\left(1+b_{h}\left|x-x_{h}\right|^{p^{\prime}}\right)^{(n-p) / p}} .
$$


Up to a subsequence, we may assume that $b_{h}$ converges to $b \in[0, \infty]$; our first goal is to check that $b \neq 0$ and $b \neq \infty$. Indeed, for any $\varepsilon>0$ there is a positive constant $\rho=\rho(\varepsilon)$ converging to 0 as $\varepsilon \rightarrow 0$ such that for any $z \in \mathbb{R}^{n}$ one has

$$
\int_{B(z, \varepsilon)}|f|^{p^{*}} \leq \rho, \quad \int_{B(0,1 / \varepsilon)}|f|^{p^{*}} \geq 1-\rho .
$$

Assume, by contradiction, that $b=\infty$. Then

$$
\int_{\left\{\left|x-x_{h}\right|>\varepsilon\right\}}\left|g_{h}(x)\right|^{p^{*}} d x \leq \varepsilon
$$

provided that $h$ is large enough depending on $\varepsilon$. Thus, for any such $h$,

$$
\begin{aligned}
& \left\|f-g_{h}\right\|_{L^{p^{*}\left(\mathbb{R}^{n}\right)}}^{p^{*}}=\left\|f-g_{h}\right\|_{L^{p^{*}\left(\left\{\left|x-x_{h}\right|>\varepsilon\right\}\right)}}^{p^{*}}+\left\|g_{h}-f\right\|_{L^{p^{*}}\left(\left\{\left|x-x_{h}\right| \leq \varepsilon\right\}\right)}^{p^{*}} \\
& \geq\left((1-\rho)^{1 / p^{*}}-\varepsilon^{1 / p^{*}}\right)^{p^{*}}+\left((1-\varepsilon)^{1 / p^{*}}-\rho^{1 / p^{*}}\right)^{p^{*}} .
\end{aligned}
$$

Passing to the limit as $h \rightarrow \infty$ in 4.7 we would get

$$
\lambda(f) \geq\left((1-\rho)^{1 / p^{*}}-\varepsilon^{1 / p^{*}}\right)^{p^{*}}+\left((1-\varepsilon)^{1 / p^{*}}-\rho^{1 / p^{*}}\right)^{p^{*}} .
$$

Hence, letting $\varepsilon \rightarrow 0$, we get $\lambda(f) \geq 2$, which contradicts the assumption. Next suppose, again by contradiction, that $b=0$. Then

$$
\int_{\{|x|<1 / \varepsilon\}}\left|g_{h}(x)\right|^{p^{*}} d x \leq \int_{\left\{\left|x-x_{h}\right|<1 / \varepsilon\right\}}\left|g_{h}(x)\right|^{p^{*}} d x \leq \varepsilon
$$

if $h$ is large enough, depending on $\varepsilon$. Analogously to 4.7, we have

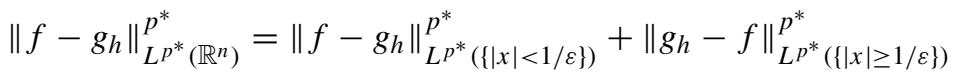

$$
\begin{aligned}
& \geq\left((1-\rho)^{1 / p^{*}}-\varepsilon^{1 / p^{*}}\right)^{p^{*}}+\left((1-\varepsilon)^{1 / p^{*}}-\rho^{1 / p^{*}}\right)^{p^{*}},
\end{aligned}
$$

and we reach the same contradiction as above.

Now, since $b_{h} \rightarrow b \in(0, \infty)$, and $\left\|g_{h}\right\|_{L^{p^{*}}\left(\mathbb{R}^{n}\right)}=1$ for every $h$, we see that $a_{h} \rightarrow a$ for some $a \in \mathbb{R} \backslash\{0\}$. Let us now show that, again up to a subsequence, there exists $\bar{x} \in \mathbb{R}^{n}$ such that $x_{h} \rightarrow \bar{x}$. In order to prove this fact, it suffices to exclude that $\left|x_{h}\right| \rightarrow \infty$. We argue by contradiction again and observe that, if this is the case, then for every $L>0$,

$$
\int_{\left\{\left|x-x_{h}\right| \leq L\right\}}|f(x)|^{p^{*}} d x \leq \frac{1}{L}
$$

if $h$ is large enough depending on $L$; for any fixed $\varepsilon>0$, since $b \in(0, \infty)$ we can choose $L$ so large that

$$
\int_{\left\{\left|x-x_{h}\right| \leq L\right\}}\left|g_{h}(x)\right|^{p^{*}} d x \geq 1-\varepsilon
$$


for every $h$. Therefore, similarly to 4.7 , we deduce that

$$
\left\|f-g_{h}\right\|_{L^{p^{*}}\left(\mathbb{R}^{n}\right)}^{p^{*}} \geq\left((1-\varepsilon)^{1 / p^{*}}-\frac{1}{L^{1 / p^{*}}}\right)^{p^{*}}+\left(\left(1-\frac{1}{L}\right)^{1 / p^{*}}-\varepsilon^{1 / p^{*}}\right)^{p^{*}},
$$

whence we get the contradiction $\lambda(f) \geq 2$ on letting $\varepsilon$ go to 0 and thus $L$ to $\infty$. Since $g_{h}$ converges to $g_{a, b, \bar{x}}$ in $L^{p^{*}}\left(\mathbb{R}^{n}\right)$, the latter function is a minimizer in the definition of $\lambda(f)$.

Step II. $\lambda(f)<2$. Set $g=g_{a, 1,0}$, where $a$ is the positive number such that $\|g\|_{L^{p^{*}}\left(\mathbb{R}^{n}\right)}$ $=1$. Set $F=\{f<g\}$ and $G=\{g<f\}$. Then

$$
|f-g|=g-f \leq g \quad \text { in } F, \quad|f-g|=f-g<f \quad \text { in } G .
$$

Note that the strict inequality above holds since $g$ is strictly positive. Thus

$$
\begin{aligned}
\lambda(f) & \leq \int_{\mathbb{R}^{n}}|f-g|^{p^{*}}=\int_{F}|f-g|^{p^{*}}+\int_{G}|f-g|^{p^{*}}<\int_{F} g p^{p^{*}}+\int_{G} f p^{p^{*}} \\
& <\int_{\mathbb{R}^{n}} g p^{p^{*}}+\int_{\mathbb{R}^{n}} f^{p^{*}}=2,
\end{aligned}
$$

and the assertion follows.

We are now ready to prove Lemma7.

Proof of Lemma 7 We may assume, without loss of generality, that $\|f\|_{L^{p^{*}}\left(\mathbb{R}^{n}\right)}=1$. Let $a, b, x_{0}$, according to Lemma 10 , be such that $\lambda(f)=\left\|f-g_{a, b, x_{0}}\right\|_{L^{p^{*}}\left(\mathbb{R}^{n}\right)}^{p^{*}}$. Denote by $z_{0}$ be the orthogonal projection of $x_{0}$ on $S$, and let $y_{0}$ be the point obtained by reflecting $x_{0}$ with respect to $S$. We have

$$
\begin{aligned}
\lambda(f \mid S)^{1 / p^{*}} & \leq\left\|f-g_{a, b, z_{0}}\right\|_{L^{p^{*}}\left(\mathbb{R}^{n}\right)} \\
& \leq\left\|f-g_{a, b, x_{0}}\right\|_{L^{p^{*}}\left(\mathbb{R}^{n}\right)}+\left\|g_{a, b, x_{0}}-g_{a, b, z_{0}}\right\|_{L^{p^{*}}\left(\mathbb{R}^{n}\right)} \\
& =\lambda(f)^{1 / p^{*}}+\left\|g_{a, b, x_{0}}-g_{a, b, z_{0}}\right\|_{L^{p^{*}}\left(\mathbb{R}^{n}\right)} .
\end{aligned}
$$

By Lemma $9 .\left\|g_{a, b, x_{0}}-g_{a, b, z_{0}}\right\|_{L^{p^{*}}\left(\mathbb{R}^{n}\right)} \leq\left\|g_{a, b, x_{0}}-g_{a, b, y_{0}}\right\|_{L^{p^{*}}\left(\mathbb{R}^{n}\right)}$. On the other hand, the symmetries of $f$ entail that $\lambda(f)=\left\|f-g_{a, b, y_{0}}\right\|_{L^{p^{*}}\left(\mathbb{R}^{n}\right)}$. Hence,

$\left\|g_{a, b, x_{0}}-g_{a, b, y_{0}}\right\|_{L^{p^{*}}\left(\mathbb{R}^{n}\right)} \leq\left\|f-g_{a, b, y_{0}}\right\|_{L^{p^{*}}\left(\mathbb{R}^{n}\right)}+\left\|g_{a, b, x_{0}}-f\right\|_{L^{p^{*}}\left(\mathbb{R}^{n}\right)}=2 \lambda(f)^{1 / p^{*}}$.

Therefore $\lambda(f \mid S)^{1 / p^{*}} \leq 3 \lambda(f)^{1 / p^{*}}$.

The contribution of Lemma 11 below is in the same direction as Lemma 7 and provides an estimate for $\lambda(f \mid H)$ in terms of $\lambda(f)$, when $H$ is a hyperplane splitting $f$ in two functions having the same $L^{p^{*}}$ norm. In what follows, we denote by $H^{+}$and $H^{-}$the two half-spaces into which $\mathbb{R}^{n}$ is split by $H$. Moreover, we denote by $T_{H}: \mathbb{R}^{n} \rightarrow \mathbb{R}^{n}$ the map which associates to any $x \in \mathbb{R}^{n}$ the point $T_{H}(x)$ obtained by reflecting $x$ with respect to $H$. 
Lemma 11. Let $f$ be any nonnegative function from $W^{1, p}\left(\mathbb{R}^{n}\right)$, and let $H$ be any hyperplane such that

$$
\int_{H^{+}} f^{p^{*}}=\int_{H^{-}} f^{p^{*}}=\frac{1}{2} \int_{\mathbb{R}^{n}} f^{p^{*}} .
$$

Then there exists a constant $C$ such that

$$
\lambda(f \mid H) \leq C \lambda(f)^{1 / p^{*}}
$$

and

$$
\int_{\mathbb{R}^{n}}\left|f \circ T_{H}-f\right|^{p^{*}} \leq C\|f\|_{L^{p^{*}}\left(\mathbb{R}^{n}\right)}^{p^{*}} \lambda(f)^{1 / p^{*}} .
$$

Proof. We may assume, without loss of generality, that $\|f\|_{L^{p^{*}}\left(\mathbb{R}^{n}\right)}=1$. By Lemma 10 . let $a, b, x_{0}$ be such that $\lambda(f)=\left\|f-g_{a, b, x_{0}}\right\|_{L^{p^{*}}\left(\mathbb{R}^{n}\right)}^{p^{*}}$, and denote $g_{a, b, x_{0}}$ simply by $g_{0}$. Let $\bar{x}$ be the projection of $x_{0}$ on $H$, and set $\bar{g}=g_{a, b, \bar{x}}$. Then

$$
\lambda(f \mid H) \leq \int_{\mathbb{R}^{n}}|f-\bar{g}|^{p^{*}} \leq 2^{p^{*}-1}\left\{\lambda(f)+\int_{\mathbb{R}^{n}}\left|g_{0}-\bar{g}\right|^{p^{*}}\right\} .
$$

Let us now consider the half-spaces $K^{ \pm}=\left(x_{0}-\bar{x}\right)+H^{ \pm}$. Clearly

$$
\frac{1}{2}=\int_{K^{ \pm}} g_{0}^{p^{*}}=\int_{H^{ \pm}} f^{p^{*}}=\int_{H^{ \pm}} \bar{g} p^{*}
$$

On interchanging $K^{+}$with $K^{-}$if necessary, we may also assume that $K^{+} \subseteq H^{+}$and $H^{-} \subseteq K^{-}$. Thus

$$
\int_{H^{-}}\left|g_{0}-\bar{g}\right|^{p^{*}}=\int_{K^{+}}\left|g_{0}-\bar{g}\right|^{p^{*}} \leq \int_{H^{+}}\left|g_{0}-\bar{g}\right|^{p^{*}},
$$

whence

$$
\int_{\mathbb{R}^{n}}\left|g_{0}-\bar{g}\right|^{p^{*}} \leq 2 \int_{H^{+}}\left|g_{0}-\bar{g}\right|^{p^{*}} .
$$

One has $g_{0}(x) \geq \bar{g}(x)$ for $x \in K^{+}$, and hence $\left|g_{0}(x)-\bar{g}(x)\right|^{p^{*}} \leq g_{0}(x)^{p^{*}}-\bar{g}(x)^{p^{*}}$ for the same values of $x$. Thus,

$$
\begin{aligned}
\int_{K^{+}}\left|g_{0}-\bar{g}\right|^{p^{*}} & \leq \int_{K^{+}} g_{0}^{p^{*}}-\int_{K^{+}} \bar{g} p^{*}=\frac{1}{2}-\int_{H^{-}} g_{0}^{p^{*}}=\int_{H^{-}} f^{p^{*}}-\int_{H^{-}} g_{0}^{p^{*}} \\
& \leq C\left(\|f\|_{L^{p^{*}}\left(H^{-}\right)}-\left\|g_{0}\right\|_{L^{p^{*}}\left(H^{-}\right)}\right) \leq C\left\|f-g_{0}\right\|_{L^{p^{*}}\left(H^{-}\right)} \\
& \leq C \lambda(f)^{1 / p^{*}}
\end{aligned}
$$

for some positive constant $C$. Note that we have made use of the fact that $\int_{K^{+}} \bar{g} p^{*}=$ $\int_{H^{-}} g_{0}^{p^{*}}$, by symmetry. On the other hand, by symmetry again,

$$
\int_{H^{+} \backslash K^{+}}\left|g_{0}-\bar{g}\right|^{p^{*}} \leq 2^{p^{*}-1} \int_{H^{+} \backslash K^{+}}\left(g_{0}^{p^{*}}+\bar{g}^{p^{*}}\right)=2^{p^{*}} \int_{H^{+} \backslash K^{+}} g_{0}^{p^{*}} .
$$


We have

$$
\int_{H^{+} \backslash K^{+}} g_{0}^{p^{*}}=\int_{H^{+}} g_{0}^{p^{*}}-\frac{1}{2}=\int_{H^{+}} g_{0}^{p^{*}}-\int_{H^{+}} f^{p^{*}},
$$

whence, as in 4.12, one gets

$$
\int_{H^{+} \backslash K^{+}} g_{0}^{p^{*}} \leq C \lambda(f)^{1 / p^{*}} .
$$

Thus, (4.8) follows from 4.10-4.13). As far as 4.9) is concerned, if $a, b$ and $\hat{x} \in H$ are chosen, thanks to Lemma 10 in such a way that

$$
\lambda(f \mid H)=\left\|f-g_{a, b, \hat{x}}\right\|_{L^{p^{*}}\left(\mathbb{R}^{n}\right)},
$$

then, by (4.8),

$$
\begin{aligned}
& \int_{H^{ \pm}}\left|f \circ T_{H}-f\right|^{p^{*}} \leq 2^{p^{*}-1}\left(\int_{H^{+}}\left|f \circ T_{H}-g_{a, b, \hat{x}}\right|^{p^{*}}+\int_{H^{+}}\left|f-g_{a, b, \hat{x}}\right|^{p^{*}}\right) \\
& =2^{p^{*}-1} \int_{\mathbb{R}^{n}}\left|f-g_{a, b, \hat{x}}\right|^{p^{*}}=2^{p^{*}-1} \lambda(f \mid H) \leq C \lambda(f)^{1 / p^{*}} .
\end{aligned}
$$

Our next result can be regarded as a qualitative version of Theorem 1 , and enables us to restrict our attention to the case where $\lambda(f)$ does not exceed some arbitrarily prescribed constant depending only on $p$ and $n$.

Lemma 12. Let $n \geq 2$ and let $1<p<n$. For every $\varepsilon>0$ there exists $\bar{\delta}>0$ such that if $f \in W^{1, p}\left(\mathbb{R}^{n}\right)$ and $\delta(f) \leq \bar{\delta}$, then $\lambda(f) \leq \varepsilon$.

Proof. Assume, by contradiction, that there exists a sequence $\left\{f_{h}\right\} \subseteq W^{1, p}\left(\mathbb{R}^{n}\right)$ such that $\lim _{h \rightarrow \infty} \delta\left(f_{h}\right)=0$ but $\lim _{h \rightarrow \infty} \lambda\left(f_{h}\right)>0$. On normalizing, if necessary, we may assume that $\left\|f_{h}\right\|_{L^{p^{*}}\left(\mathbb{R}^{n}\right)}=1$ for every $h \in \mathbb{N}$. Since $\lim _{h \rightarrow \infty}\left\|\nabla f_{h}\right\|_{L^{p^{*}}\left(\mathbb{R}^{n}\right)}=S(p, n)$, the concentration-compactness method of Lions ([Li] $)$ can be applied (as in $[\overline{\mathrm{St}}])$ to show that there exists a subsequence of rescaled-translated functions $\tilde{f}_{h}(x)=r_{h}^{n / p^{*}} f_{h}\left(r_{h}\left(x-x_{h}\right)\right)$ such that $\tilde{f}_{h} \rightarrow f$ strongly in $L^{p^{*}}\left(\mathbb{R}^{n}\right)$ for some $f \in W^{1, p}\left(\mathbb{R}^{n}\right)$. Notice that $\lambda\left(\tilde{f}_{h}\right)=$ $\lambda\left(f_{h}\right), \delta\left(\tilde{f}_{h}\right)=\delta\left(f_{h}\right)$ and the functional $f \mapsto \lambda(f)$ is strongly continuous on $L^{p^{*}}\left(\mathbb{R}^{n}\right)$. Hence, $\lambda(f)=\lim _{h \rightarrow \infty} \lambda\left(f_{h}\right)>0$. On the other hand, by lower semicontinuity, $0=$ $\lim _{h \rightarrow \infty} \delta\left(f_{h}\right) \geq \delta(f)$, that is, $\delta(f)=0$. Consequently, since the functions in (1.2) are the only optimal functions in $[1.1]$ as proved in $[\mathrm{CNV}]$, one obtains $\lambda(f)=0$, a contradiction.

Before going into the details of the proof of Theorem 6, we briefly outline it. A preliminary step consists in associating with any function $f$ another function $\tilde{f}$ symmetric about a hyperplane, with the same $L^{p^{*}}$ norm as $f$, and such that $\lambda(f) \leq C \lambda(\tilde{f})$ and $\delta(\widetilde{f}) \leq 2 \delta(f)$. To this end, a first natural attempt could consist in selecting a hyperplane $H$ in $\mathbb{R}^{n}$ having the property that the restrictions of $f$ to the two half-spaces bounded by $H$ have equal $L^{p^{*}}$ norms, and then, by reflecting these two restrictions, to define two candidates $\widetilde{f}$ which are symmetric about $H$. Via this construction, one certainly has 
$\delta(\tilde{f}) \leq 2 \delta(f)$ for both choices of $\tilde{f}$, but, unfortunately, the inequality $\lambda(f) \leq C \lambda(\tilde{f})$ may fail for both of them, whatever $C$ is. To see this, consider for instance the hyperplane $H=\left\{x_{1}=0\right\}$ and the function $f$ given by

$$
f(x)= \begin{cases}g_{a^{\prime}, b^{\prime}, 0}(x) & \text { if } x_{1} \leq 0, \\ g_{a^{\prime \prime}, b^{\prime \prime}, 0}(x) & \text { otherwise, }\end{cases}
$$

where $g_{a, b, x_{0}}$ are the extremal functions defined in 1.2 , and $a^{\prime} \neq a^{\prime \prime}$ and $b^{\prime} \neq b^{\prime \prime}$ are chosen in such a way that $\int_{\left\{x_{1} \_0\right\}} f^{p^{*}}=\int_{\left\{x_{1}>0\right\}} f^{p^{*}}$. On reflecting $f$ with respect to $H$ one has either $\tilde{f}=g_{a^{\prime}, b^{\prime}, 0}$, or $\widetilde{f}=g_{a^{\prime \prime}, b^{\prime \prime}, 0}$, and hence $\lambda(\tilde{f})=0<\lambda(f)$. Note, however, that this is not the case if $f$ is reflected with respect to a hyperplane orthogonal to $H$. This observation gives a hint on how to repair this shortcoming. Indeed, one can prove that if $f$ and $H$ are such that $C \lambda(\tilde{f})<\lambda(f)$ for a sufficiently large constant $C=C(n, p)$, then $f$ and $H$ are nearly in the situation of (4.14), and one can show that the functions $\tilde{f}$ constructed by reflecting $f$ with respect to any hyperplane $H^{\prime}$ orthogonal to $H$ actually satisfy $\lambda(f) \leq C \lambda(\widetilde{f})$. Thus, a function $\widetilde{f}$ symmetric about a hyperplane and fulfilling our requirements can always be constructed.

An iteration of this construction produces an $(n-1)$-symmetric function still satisfying these requirements. This is the content of Lemma 13 below. However, this argument cannot be applied as a final step to obtain an $n$-symmetric function. Indeed, in order to preserve the symmetries already achieved, at any step the two orthogonal hyperplanes between which we choose have to be orthogonal to all the hyperplanes selected at the previous steps. But once $n-1$ hyperplanes of symmetry have been fixed, no room is left to choose two more orthogonal hyperplanes. Due to this problem, the accomplishment of the last symmetry requires a more delicate argument. Such an argument also relies upon Corollary 4, and its use, which seems indispensable at this stage, explains the presence of the exponent $1 / \beta p^{*}$ in estimate (4.1). We start from a function $f$ which is already symmetric about $n-1$ orthogonal hyperplanes, one of which we call $H$. Consider an hyperplane $\widetilde{H}$ orthogonal to the $n-1$ hyperplanes of symmetry of $f$, and such that the restrictions of $f$ to the two half-spaces bounded by $H$ have equal $L^{p^{*}}$ norms. If one of the two functions $\widetilde{f}$ obtained on reflecting these restrictions with respect to $\widetilde{H}$ has the property that $\lambda(f) \leq C \lambda(\widetilde{f})$, then we are done. Otherwise, we already know that $f$ is nearly as in 4.14. We then consider the two orthogonal hyperplanes $H^{\prime}$ and $H^{\prime \prime}$ such that the angles between $H^{\prime}$ and $H$ and between $H^{\prime \prime}$ and $\widetilde{H}$ have aperture $\pi / 4$, and moreover $H^{\prime} \cap H^{\prime \prime}=H \cap \widetilde{H}$, up to translations. Since the function $f$ is far from having the form 4.14) both with $H=H^{\prime}$ and with $H=H^{\prime \prime}$, a double reflection argument with respect to $H^{\prime}$ and $H^{\prime \prime}$ yields a function $\widetilde{f}$ fulfilling $\lambda(f) \leq C \lambda(\widetilde{f})$. Furthermore, since $H^{\prime}$ and $H^{\prime \prime}$ are orthogonal to the first $n-2$ hyperplanes, the first $n-2$ symmetries of $f$ are preserved for $\widetilde{f}$. The symmetry about $H$ is obviously lost, but $\widetilde{f}$ is symmetric both about $H^{\prime}$ and about $H^{\prime \prime}$, and hence $\widetilde{f}$ is actually $n$-symmetric.

Lemma 13. Let $n \geq 2$ and let $1<p<n$. Then there exists a positive constant $C$ having the following property. For every nonnegative function $f \in W^{1, p}\left(\mathbb{R}^{n}\right)$ there exists an (n-1)-symmetric function $\tilde{f} \in W^{1, p}\left(\mathbb{R}^{n}\right)$ such that

$$
\lambda(f) \leq C \lambda(\tilde{f}), \quad \delta(\tilde{f}) \leq 2^{n-1} \delta(f) .
$$


Proof. As usual, we may assume that $\|f\|_{L^{p^{*}}\left(\mathbb{R}^{n}\right)}=1$. Moreover we may suppose that $\delta(f) \leq \bar{\delta}$ for some positive constant $\bar{\delta}$ (depending only on $p$ and $n$ ) to be chosen later. Indeed, if $\delta(f) \geq \bar{\delta}$, one can pick a spherically symmetric function $g$, independent of $f$, such that $0<\delta(g) \leq 2^{n-1} \bar{\delta}$. Thus $\delta(g) \leq 2^{n-1} \delta(f)$, and $\lambda(f) \leq 2^{p^{*}} \leq$ $\left(2^{p^{*}} / \lambda(g)\right) \lambda(g) \leq C \lambda(g)$, and hence the first inequality in 4.15 is fulfilled with $\widetilde{f}=\bar{g}$.

If $\delta(f) \leq \bar{\delta}$, fixing any coordinate direction $e_{k}$ with $1 \leq k \leq n$, consider a hyperplane $H_{k}$ orthogonal to $e_{k}$ and the corresponding half-spaces $H_{k}^{+}$and $H_{k}^{-}$, having the property that

$$
\int_{H_{k}^{+}} f^{p^{*}}=\int_{H_{k}^{-}} f^{p^{*}}=\frac{1}{2}
$$

For simplicity, denote by $T_{k}: \mathbb{R}^{n} \rightarrow \mathbb{R}^{n}$ the reflection $T_{H_{k}}$ with respect to $H_{k}$, and define

$$
f_{k}^{+}(x)=\left\{\begin{array}{ll}
f(x) & \text { if } x \in H_{k}^{+}, \\
f\left(T_{k}(x)\right) & \text { if } x \in H_{k}^{-} ;
\end{array} \quad f_{k}^{-}(x)= \begin{cases}f\left(T_{k}(x)\right) & \text { if } x \in H_{k}^{+}, \\
f(x) & \text { if } x \in H_{k}^{-}\end{cases}\right.
$$

Clearly, $f_{k}^{ \pm}$are nonnegative functions from $W^{1, p}\left(\mathbb{R}^{n}\right)$, symmetric about $H_{k}$, and satisfying $\left\|f_{k}^{ \pm}\right\|_{L^{p^{*}\left(\mathbb{R}^{n}\right)}}=1$. Moreover,

$$
\begin{aligned}
\|\nabla f\|_{L^{p}\left(\mathbb{R}^{n}\right)} & =\left(\int_{H_{k}^{+}}\left|\nabla f_{k}^{+}\right|^{p}+\int_{H_{k}^{-}}\left|\nabla f_{k}^{-}\right|^{p}\right)^{1 / p} \\
& =\left(\frac{1}{2} \int_{\mathbb{R}^{n}}\left|\nabla f_{k}^{+}\right|^{p}+\frac{1}{2} \int_{\mathbb{R}^{n}}\left|\nabla f_{k}^{-}\right|^{p}\right)^{1 / p} \\
& \geq \frac{1}{2}\left(\left\|\nabla f_{k}^{+}\right\|_{L^{p}\left(\mathbb{R}^{n}\right)}+\left\|\nabla f_{k}^{-}\right\|_{L^{p}\left(\mathbb{R}^{n}\right)}\right) .
\end{aligned}
$$

In particular,

$$
\max \left\{\delta\left(f_{k}^{+}\right), \delta\left(f_{k}^{-}\right)\right\} \leq 2 \delta(f) .
$$

Denote by $g_{k}^{+}$and $g_{k}^{-}$two functions realizing the minima in $\lambda\left(f_{k}^{ \pm} \mid H_{k}\right)$, again thanks to Lemma10, Then

$$
\begin{aligned}
\lambda(f) & \leq \int_{\mathbb{R}^{n}}\left|f-g_{k}^{+}\right|^{p^{*}}=\int_{H_{k}^{+}}\left|f_{k}^{+}-g_{k}^{+}\right|^{p^{*}}+\int_{H_{k}^{-}}\left|f_{k}^{-}-g_{k}^{+}\right|^{p^{*}} \\
& \leq 2^{p^{*}-1}\left(\frac{\lambda\left(f_{k}^{+} \mid H_{k}\right)+\lambda\left(f_{k}^{-} \mid H_{k}\right)}{2}+\int_{H_{k}^{-}}\left|g_{k}^{+}-g_{k}^{-}\right|^{p^{*}}\right) \\
& \leq 2^{p^{*}-2} 3^{p^{*}}\left(\lambda\left(f_{k}^{+}\right)+\lambda\left(f_{k}^{-}\right)+\int_{H_{k}^{-}}\left|g_{k}^{+}-g_{k}^{-}\right|^{p^{*}}\right) .
\end{aligned}
$$

Note that, in the last inequality, we have applied Lemma 7 to $f_{k}^{ \pm}$. Now, we claim that there exist positive constants $C$ and $\bar{\delta}$ having the following property: whenever $\delta(f) \leq \bar{\delta}$ and $1 \leq i<j \leq n$, there exists $k \in\{i, j\}$ such that

$$
\int_{H_{k}^{-}}\left|g_{k}^{+}-g_{k}^{-}\right|^{p^{*}} \leq C\left(\int_{H_{k}^{+}}\left|f_{k}^{+}-g_{k}^{+}\right|^{p^{*}}+\int_{H_{k}^{-}}\left|f_{k}^{-}-g_{k}^{-}\right|^{p^{*}}\right) .
$$


Observe that once this claim is established, 4.15 will follow. Indeed, take $i=1$ and $j=2$ and suppose (up to relabelling the indices) that 4.19) holds with $k=1$. Then from (4.18) and 4.17) applied with $k=1$, we infer that

$$
\lambda(f) \leq C^{\prime}\left(\lambda\left(f_{1}^{+}\right)+\lambda\left(f_{1}^{-}\right)\right), \quad \max \left\{\delta\left(f_{1}^{+}\right), \delta\left(f_{1}^{-}\right)\right\} \leq 2 \delta(f)
$$

for some constant $C^{\prime}$. In particular, at least one of the functions $f_{1}^{ \pm}$, denote it by $f_{1}$, satisfies $\lambda(f) \leq 2 C^{\prime} \lambda\left(f_{1}\right)$ and $\delta\left(f_{1}\right) \leq 2 \delta(f)$. Moreover, $f_{1}$ is 1 -symmetric and satisfies $\left\|f_{1}\right\|_{L^{p^{*}}\left(\mathbb{R}^{n}\right)}=1$. Then one can repeat the argument starting from $f_{1}$, and obtain a 2 symmetric function $f_{2}$ fulfilling $\lambda(f) \leq 4 C^{\prime 2} \lambda\left(f_{2}\right)$ and $\delta\left(f_{2}\right) \leq 4 \delta(f)$. On iterating this procedure, 4.15 follows.

We now have to prove our claim. The crucial observation is that, when $\delta$ is sufficiently small, all the functions $g_{k}^{ \pm}, 1 \leq k \leq n$, are close to each other in the $L^{p^{*}}$ norm, in the sense that there is a constant $C_{0}$ such that

$$
\int_{\mathbb{R}^{n}}\left|g_{i}^{\sigma}-g_{j}^{\tau}\right|^{p^{*}} \leq C_{0} \int_{H_{i}^{\sigma} \cap H_{j}^{\tau}}\left|g_{i}^{\sigma}-g_{j}^{\tau}\right|^{p^{*}} \quad \text { for every } 1 \leq i<j \leq n, \sigma, \tau \in\{+,-\} .
$$

To verify (4.20), let us begin by noting that there exist constants $\varrho$ and $C_{1}$ such that if

(i) $\int_{\mathbb{R}^{n}} g_{a, b, x_{0}}^{p^{*}}=\int_{\mathbb{R}^{n}} g_{c, d, y_{0}}^{p^{*}}=1$,

(ii) $I$ and $J$ are two orthogonal half-spaces with $x_{0} \in \partial I$ and $y_{0} \in \partial J$,

(iii) $\int_{I \cap J} g_{a, b, x_{0}}^{p^{*}} \geq 1 / 8$ and $\int_{I \cap J} g_{c, d, y_{0}}^{p^{*}} \geq 1 / 8$,

(iv) $\int_{\mathbb{R}^{n}}\left|g_{a, b, x_{0}}-g_{c, d, y_{0}}\right|^{p^{*}} \leq \varrho$,

then

$$
\int_{\mathbb{R}^{n}}\left|g_{a, b, x_{0}}-g_{c, d, y_{0} \mid}\right|^{p^{*}} \leq C_{1} \int_{I \cap J}\left|g_{a, b, x_{0}}-g_{c, d, y_{0}}\right|^{p^{*}} .
$$

Since 4.20 is a consequence of this assertion with the choice $g_{a, b, x_{0}}=g_{i}^{\sigma}, g_{c, d, y_{0}}=g_{j}^{\tau}$, $I=H_{i}^{\sigma}$ and $J=H_{j}^{\tau}$ we only have to check that (i)-(iv) are fulfilled in the present situation.

Properties (i), (ii) hold by construction. The choice of $\bar{\delta}$ comes into play in connection with conditions (iii) and (iv). Actually condition (iii) is easily seen to hold provided that $\lambda(f)$ is sufficiently small, and we may suppose that this is the case, thanks to Lemma 12 . since we are assuming that $\delta(f) \leq \bar{\delta}$. Inequality (iv) relies upon Lemmas 11 and 12 Indeed,

$$
\begin{gathered}
\left\|g_{i}^{\sigma}-g_{j}^{\tau}\right\|_{L^{p^{*}}\left(\mathbb{R}^{n}\right)} \leq \\
\left\|g_{i}^{\sigma}-f_{i}^{\sigma}\right\|_{L^{p^{*}}\left(\mathbb{R}^{n}\right)}+\left\|f_{i}^{\sigma}-f\right\|_{L^{p^{*}}\left(\mathbb{R}^{n}\right)}+\left\|f-f_{j}^{\tau}\right\|_{L^{p^{*}}\left(\mathbb{R}^{n}\right)} \\
+\left\|f_{j}^{\tau}-g_{j}^{\tau}\right\|_{L^{p^{*}}\left(\mathbb{R}^{n}\right) .}
\end{gathered}
$$

By 4.9),

$$
\int_{\mathbb{R}^{n}}\left|f_{i}^{\sigma}-f\right|^{p^{*}}=\frac{1}{2} \int_{\mathbb{R}^{n}}\left|f \circ T_{i}-f\right|^{p^{*}} \leq C \lambda(f)^{1 / p^{*}}
$$


and hence the second and the third norm on the right-hand side of 4.21 can be made arbitrarily small, owing to Lemma 12 , by a suitable choice of $\bar{\delta}$. The same assertion holds also for the other two norms, inasmuch as

$$
\int_{\mathbb{R}^{n}}\left|g_{i}^{\sigma}-f_{i}^{\sigma}\right|^{p^{*}} \leq 2^{p^{*}-1}\left(\lambda\left(f \mid H_{i}\right)+\left\|f-f_{i}^{\sigma}\right\|_{L^{p^{*}}\left(\mathbb{R}^{n}\right)}^{p^{*}}\right) \leq C \lambda(f)^{1 / p^{*}}
$$

for some constant $C$, by (4.8). Inequality (4.20) is thus established. It only remains to make use of (4.20) to prove the claim concerning (4.19). To fix ideas, suppose that $i=1$ and $j=2$. Set, for $k=1,2$,

$$
h_{k}=g_{k}^{+} \chi_{H_{k}^{+}}+g_{k}^{-} \chi_{H_{k}^{-}}
$$

By 4.20, ,

$\int_{\mathbb{R}^{n}}\left|h_{1}-h_{2}\right|^{p^{*}} \geq \int_{H_{1}^{+} \cap H_{2}^{+}}\left|h_{1}-h_{2}\right|^{p^{*}}=\int_{H_{1}^{+} \cap H_{2}^{+}}\left|g_{1}^{+}-g_{2}^{+}\right|^{p^{*}} \geq \frac{1}{C_{0}} \int_{\mathbb{R}^{n}}\left|g_{1}^{+}-g_{2}^{+}\right|^{p^{*}}$.

A similar chain with $H_{1}^{+} \cap H_{2}^{+}$replaced by $H_{1}^{-} \cap H_{2}^{+}$yields

$$
\int_{\mathbb{R}^{n}}\left|h_{1}-h_{2}\right|^{p^{*}} \geq \frac{1}{C_{0}} \int_{\mathbb{R}^{n}}\left|g_{1}^{-}-g_{2}^{+}\right|^{p^{*}} .
$$

In conclusion,

$$
\int_{\mathbb{R}^{n}}\left|g_{1}^{+}-g_{1}^{-}\right|^{p^{*}} \leq 2^{p^{*}-1} C_{0} \int_{\mathbb{R}^{n}}\left|h_{1}-h_{2}\right|^{p^{*}}
$$

An analogous argument tells us that

$$
\int_{\mathbb{R}^{n}}\left|g_{2}^{+}-g_{2}^{-}\right|^{p^{*}} \leq 2^{p^{*}-1} C_{0} \int_{\mathbb{R}^{n}}\left|h_{1}-h_{2}\right|^{p^{*}}
$$

On the other hand,

$$
\begin{aligned}
\int_{\mathbb{R}^{n}}\left|h_{1}-h_{2}\right|^{p^{*}} \leq & 2^{p^{*}-1}\left(\int_{\mathbb{R}^{n}}\left|h_{1}-f\right|^{p^{*}}+\int_{\mathbb{R}^{n}}\left|h_{2}-f\right|^{p^{*}}\right) \\
= & 2^{p^{*}-1}\left(\int_{H_{1}^{+}}\left|g_{1}^{+}-f_{1}^{+}\right|^{p^{*}}+\int_{H_{1}^{-}}\left|g_{1}^{-}-f_{1}^{-}\right|^{p^{*}}\right. \\
& \left.\quad+\int_{H_{2}^{+}}\left|g_{2}^{+}-f_{2}^{+}\right|^{p^{*}}+\int_{H_{2}^{-}}\left|g_{2}^{-}-f_{2}^{-}\right|^{p^{*}}\right) .
\end{aligned}
$$

Combining (4.22 -4.23 ensures that 4.19 holds for an appropriate constant $C$, with either $k=1$ or $k=2$. 
Proof of Theorem 6. We may assume that $\|f\|_{L^{p^{*}}\left(\mathbb{R}^{n}\right)}=1$ and, by Lemma 13 , that $f$ is $(n-1)$-symmetric. As in the proof of that lemma, we may also suppose that $\delta(f)$ does not exceed a constant $\bar{\delta}$ to be chosen later. Finally, up to an isometry, we may assume that $f$ is symmetric about the last $n-1$ coordinate hyperplanes and that

$$
\int_{\left\{x_{1}>0\right\}} f^{p^{*}}=\frac{1}{2}
$$

Let $f^{+}$and $f^{-}$be defined as in (4.16), with $H_{1}=\left\{x_{1}=0\right\}$; denote by $C_{0}$ a (sufficiently large) positive constant to be chosen later. By (4.17),

$$
\max \left\{\delta\left(f^{+}\right), \delta\left(f^{-}\right)\right\} \leq 2 \delta(f) .
$$

Thus, if either $\lambda(f) \leq C_{0} \lambda\left(f^{+}\right)$, or $\lambda(f) \leq C_{0} \lambda\left(f^{-}\right)$, inequality (4.1) immediately follows, with either $\widehat{f}=f^{+}$or $\widehat{f}=f^{-}$, since both $f^{+}$and $f^{-}$are $n$-symmetric. Therefore, we may focus on the case where

$$
\lambda(f) \geq C_{0} \max \left\{\lambda\left(f^{+}\right), \lambda\left(f^{-}\right)\right\} .
$$

Consider the set $Q=\left\{x \in \mathbb{R}^{n}:\left|x_{1}\right| \leq x_{2}\right\}$ and define the function $\widehat{f}: \mathbb{R}^{n} \rightarrow[0, \infty)$ as

$$
\widehat{f}(x)= \begin{cases}f(x) & \text { if } x \in Q, \\ f\left(R_{1}(x)\right) & \text { if } x \in R_{1}(Q), \\ \widehat{f}\left(R_{2}(x)\right) & \text { if } x \in R_{2}\left(Q \cup R_{1}(Q)\right),\end{cases}
$$

where $R_{1}, R_{2}: \mathbb{R}^{n} \rightarrow \mathbb{R}^{n}$ denote the reflections with respect to the hyperplanes $\{x \in$ $\left.\mathbb{R}^{n}: x_{2}=x_{1}\right\}$ and $\left\{x \in \mathbb{R}^{n}: x_{2}=-x_{1}\right\}$, respectively; notice that $\widehat{f}$ is symmetric with respect to the hyperplanes $\left\{x_{1}= \pm x_{2}\right\}$ and $\left\{x_{i}=0\right\}$ for $3 \leq i \leq n$, hence $n$-symmetric. Moreover, on setting $Q^{+}=\left\{x \in Q: x_{1}>0\right\}$ and $Q^{-}=\left\{x \in Q: x_{1}<0\right\}$, one has

$$
\widehat{f}=f^{+} \text {in } Q^{+}, \quad \widehat{f}=f^{-} \text {in } Q^{-} \text {. }
$$

We claim that if $C_{0}$ is sufficiently large, then there exists a constant $C$ such that (4.1) holds. Let us begin by proving the first inequality: obviously $\int_{\mathbb{R}^{n}} \widehat{f} p^{*} \leq 2$ so that, on denoting by $\widehat{g}, g^{+}$and $g^{-}$functions having the form (1.2), at which the infima in the definitions of $\lambda(\widehat{f} \mid\{0\}), \lambda\left(f^{+} \mid\{0\}\right)$ and $\lambda\left(f^{-} \mid\{0\}\right)$ are attained, we have

$$
\begin{aligned}
3^{p^{*}} \lambda(\widehat{f}) & \geq \lambda(\widehat{f} \mid\{0\})=\frac{\int_{\mathbb{R}^{n}}|\widehat{f}-\widehat{g}|^{p^{*}}}{\int_{\mathbb{R}^{n}} \widehat{f} p^{*}}=4 \frac{\int_{Q}|f-\widehat{g}|^{p^{*}}}{\int_{\mathbb{R}^{n}} \widehat{f} p^{*}} \\
& \geq 2\left(\int_{Q^{+}}\left|f^{+}-\widehat{g}\right|^{p^{*}}+\int_{Q^{-}}\left|f^{-}-\widehat{g}\right|^{p^{*}}\right) \\
& =2\left(\int_{Q^{+}}\left|f^{+}-\widehat{g}\right|^{p^{*}}+\int_{Q^{+}}\left|f^{-}-\widehat{g}\right|^{p^{*}}\right) \geq \frac{1}{2 p^{*-2}} \int_{Q^{+}}\left|f^{+}-f^{-}\right|^{p^{*}} .
\end{aligned}
$$

Observe that the first inequality holds thanks to Lemma 7. We are going to show that

$$
\int_{Q^{+}}\left|f^{+}-f^{-}\right|^{p^{*}} \geq \frac{\lambda(f)}{4 p^{*}+2},
$$


provided that $C_{0}$ is large enough, whence the first inequality in 4.1 follows. One has

$$
\begin{aligned}
\| f^{+}- & f^{-}\left\|_{L^{p^{*}}\left(Q^{+}\right)} \geq \frac{1}{2}\right\| f^{+}-f^{-} \|_{L^{p^{*}}(Q)} \\
& \geq \frac{1}{2}\left(\left\|g^{+}-g^{-}\right\|_{L^{p^{*}}(Q)}-\left\|f^{+}-g^{+}\right\|_{L^{p^{*}}(Q)}-\left\|f^{-}-g^{-}\right\|_{L^{p^{*}}(Q)}\right) .
\end{aligned}
$$

Moreover,

$$
\int_{Q}\left|f^{ \pm}-g^{ \pm}\right|^{p^{*}} \leq \int_{\mathbb{R}^{n}}\left|f^{ \pm}-g^{ \pm}\right|^{p^{*}}=\lambda\left(f^{ \pm} \mid\{0\}\right) \leq 3^{p^{*}} \lambda\left(f^{ \pm}\right) \leq \frac{3^{p^{*}}}{C_{0}} \lambda(f),
$$

where we have exploited Lemma 7 and (4.24). From 4.26) and 4.27) we get

$$
\left\|f^{+}-f^{-}\right\|_{L^{p^{*}}\left(Q^{+}\right)} \geq \frac{1}{2}\left(\left\|g^{+}-g^{-}\right\|_{L^{p^{*}}(Q)}-2\left(\frac{3^{p^{*}}}{C_{0}} \lambda(f)\right)^{1 / p^{*}}\right) .
$$

On the other hand, owing to 4.27,

$$
\begin{aligned}
\lambda(f) & \leq \int_{\mathbb{R}^{n}}\left|f-g^{+}\right| p^{*}=\frac{1}{2} \int_{\mathbb{R}^{n}}\left|g^{+}-f^{+}\right|^{p^{*}}+\frac{1}{2} \int_{\mathbb{R}^{n}}\left|g^{+}-f^{-}\right|^{p^{*}} \\
& \leq \frac{3^{p^{*}}}{2 C_{0}} \lambda(f)+2^{p^{*}-2}\left(\frac{3^{p^{*}}}{C_{0}} \lambda(f)+\int_{\mathbb{R}^{n}}\left|g^{+}-g^{-}\right|^{p^{*}}\right) .
\end{aligned}
$$

Thus,

$$
\int_{\mathbb{R}^{n}}\left|g^{+}-g^{-}\right|^{p^{*}} \geq \frac{1}{2^{p^{*}-2}}\left(1-\frac{3^{p^{*}}}{C_{0}}\left(\frac{1}{2}+2^{p^{*}-2}\right)\right) \lambda(f) \geq \frac{1}{2^{p^{*}}} \lambda(f)
$$

if $C_{0}$ is sufficiently large. Coupling (4.28) and 4.29) yields 4.25, provided that $C_{0}$ is large enough.

Let us now prove the second inequality in 4.1. One has

$$
\left|\|f\|_{L^{p^{*}}\left(Q^{+}\right)}-\frac{1}{8^{1 / p^{*}}}\right|=\left|\left\|f^{+}\right\|_{L^{p^{*}}\left(Q^{+}\right)}-\left\|g^{+}\right\|_{L^{p^{*}}\left(Q^{+}\right)}\right| \leq\left\|f^{+}-g^{+}\right\|_{L^{p^{*}}\left(Q^{+}\right)} .
$$

Since there exists a constant $C$ such that $\left|s^{p^{*}}-r p^{*}\right| \leq C|s-r|$ for $r, s \in[0,1]$, we have

$$
\begin{aligned}
\left|\int_{Q^{+}} f^{p^{*}}-\frac{1}{8}\right| & \leq C\left\|f^{+}-g^{+}\right\|_{L^{p^{*}}\left(Q^{+}\right)} \leq C \lambda\left(f^{+} \mid\{0\}\right)^{1 / p^{*}} \leq C \lambda\left(f^{+}\right)^{1 / p^{*}} \\
& \leq C \delta\left(f^{+}\right)^{1 / \beta p^{*}} \leq C \delta(f)^{1 / \beta p^{*}}
\end{aligned}
$$

for a suitable constant $C$. Note that the third inequality relies on Lemma7 7 , whereas Corollary 4 plays a role in the fourth one. The same estimate also holds in $Q^{-}, U^{+}=\left\{x_{2}>0\right.$, $\left.x_{1}>0\right\} \backslash Q$ and $U^{-}=\left\{x_{2}>0, x_{1}<0\right\} \backslash Q$. As a consequence,

$$
\left|\int_{\mathbb{R}^{n}} \widehat{f}^{p^{*}}-1\right| \leq C \delta(f)^{1 / \beta p^{*}}
$$


As far as the gradient of $\widehat{f}$ is concerned, we obviously have

$$
\int_{\mathbb{R}^{n}}|\nabla \widehat{f \mid}|^{p}=4 \int_{Q} \mid \nabla \widehat{f}^{p}=4\left(\int_{\left\{x_{2}>0\right\}}|\nabla f|^{p}-\int_{U^{+} \cup U^{-}}|\nabla f|^{p}\right) .
$$

Since $f$ is symmetric about the hyperplane $\left\{x_{2}=0\right\}$,

$$
\int_{\left\{x_{2}>0\right\}}|\nabla f|^{p}=\frac{1}{2} \int_{\mathbb{R}^{n}}|\nabla f|^{p}=S(p, n)^{p} \frac{(1+\delta(f))^{p}}{2} \leq S(p, n)^{p} \frac{(1+C \delta(f))}{2} .
$$

Here, we have made use of the fact that $\delta(f) \leq \bar{\delta}$. Applying the Sobolev inequality 1.1 to the function obtained by reflecting $f_{\mid U^{+}}$first with respect to $\left\{x_{2}=x_{1}\right\}$, then with respect to $\left\{x_{1}=0\right\}$, and finally with respect to $\left\{x_{2}=0\right\}$, and keeping in mind 4.30, we have

$$
\begin{aligned}
\int_{U^{+}}|\nabla f|^{p} & \geq 8^{-1+p / p^{*}} S(p, n)^{p}\left(\int_{U^{+}} f^{p^{*}}\right)^{p / p^{*}} \\
& \geq 8^{-1+p / p^{*}} S(p, n)^{p}\left(\frac{1}{8}-C \delta(f)^{1 / \beta p^{*}}\right)^{p / p^{*}} \\
& \geq S(p, n)^{p}\left(\frac{1}{8}-C \delta(f)^{1 / \beta p^{*}}\right),
\end{aligned}
$$

provided that $\bar{\delta}$ is small enough. An analogous estimate holds for $\int_{U^{-}}|\nabla f|^{p}$. Combining these estimates with 4.32 and 4.33) tells us that

$$
\begin{aligned}
\int_{\mathbb{R}^{n}}|\nabla \widehat{f}|^{p} & \leq 4\left(\frac{S(p, n)^{p}(1+C \delta(f))}{2}-2 S(p, n)^{p}\left(\frac{1}{8}-C \delta(f)^{1 / \beta p^{*}}\right)\right) \\
& \leq S(p, n)^{p}\left(1+C \delta(f)^{1 / \beta p^{*}}\right) .
\end{aligned}
$$

Therefore, from 4.31 we conclude that

$$
\delta(\widehat{f})=\frac{\|\nabla \widehat{f}\|_{L^{p}\left(\mathbb{R}^{n}\right.}}{S(p, n)\|\widehat{f}\|_{L^{p^{*}}\left(\mathbb{R}^{n}\right)}}-1 \leq \frac{S(p, n)\left(1+C \delta(f)^{1 / \beta p^{*}}\right)}{S(p, n)\left(1-C \delta(f)^{\left.1 / \beta p^{*}\right)}\right.}-1 \leq C \delta(f)^{1 / \beta p^{*}},
$$

which is the second inequality in (4.1).

\section{References}

[Au] Aubin, T.: Problèmes isopérimétriques et espaces de Sobolev. J. Differential Geom. 11, 573-598 (1976) Zbl 0371.46011 MR 0448404

[BWW] Bartsch, T., Weth, T., Willem, M.: A Sobolev inequality with remainder term and critical equations on domains with topology for the polyharmonic operator. Calc. Var. Partial Differential Equations 18, 253-268 (2003) Zbl 1059.31006 MR 2018667

[BE] Bianchi, G., Egnell, H.: A note on the Sobolev inequality. J. Funct. Anal. 100, 18-24 (1991) Zbl 0755.46014 MR 1124290

[Bl] Bliss, G. A.: An integral inequality. J. London Math. Soc. 5, 40-46 (1930) JFM 56.0434.02 
[BL] Brezis, H., Lieb, E. H.: Sobolev inequalities with remainder terms. J. Funct. Anal. 62, 73-86 (1985) Zbl 0577.46031 MR 0790771

[BZ] Brothers, J. E., Ziemer, W. P.: Minimal rearrangements of Sobolev functions. J. Reine Angew. Math. 384, 153-179 (1988) Zbl 0633.46030 MR 0929981

[Ci1] Cianchi, A.: A quantitative Sobolev inequality in BV. J. Funct. Anal. 237, 466-481 (2006) Zbl 1110.46020 MR 2230346

[Ci2] Cianchi, A.: Sharp Sobolev-Morrey inequalities and the distance from extremals. Trans. Amer. Math. Soc. 360, 4335-4347 (2008) Zbl 1153.46019 MR 2395175

[CEFT] Cianchi, A., Esposito, L., Fusco, N., Trombetti, C.: A quantitative Pólya-Szegö principle. J. Reine Angew. Math. 614, 153-189 (2008) Zbl pre05267828 MR 2376285

[CF1] Cianchi, A., Fusco, N.: Functions of bounded variation and rearrangements. Arch. Ration. Mech. Anal. 165, 1-40 (2002) Zbl 1028.49035 MR 1947097

[CF2] Cianchi, A., Fusco, N.: Dirichlet integrals and Steiner asymmetry. Bull. Sci. Math. 130, 675-696 (2006) Zbl 1124.46017 MR 2276198

[CNV] Cordero-Erausquin, D., Nazaret, B., Villani, C.: A mass-transportation approach to sharp Sobolev and Gagliardo-Nirenberg inequalities. Adv. Math. 182, 307-332 (2004) Zbl 1048.26010 MR 2032031

[Fu] Fuglede, B.: Stability in the isoperimetric problem for convex or nearly spherical domains in $\mathbb{R}^{n}$. Trans. Amer. Math. Soc. 314, 619-638 (1989) Zbl 0679.52007 MR 0942426

[FMP1] Fusco, N., Maggi, F., Pratelli, A.: The sharp quantitative isoperimetric inequality. Ann. of Math. 168, 941-980 (2008) MR 2456887

[FMP2] Fusco, N., Maggi, F., Pratelli, A.: The sharp quantitative Sobolev inequality for functions of bounded variation. J. Funct. Anal. 244, 315-341 (2007) Zbl 1121.46029 MR 2294486

[FMP3] Fusco, N., Maggi, F., Pratelli, A.: Stability estimates for certain Faber-Krahn and isocapacitary inequalities. Ann. Scuola Norm. Sup., to appear

[GNN] Gidas, B., Ni, W. M., Nirenberg, L.: Symmetry and related properties via the maximum principle. Comm. Math. Phys. 68, 209-243 (1979) Zbl 0425.35020 MR 0544879

[Ha] Hall, R. R.: A quantitative isoperimetric inequality in $n$-dimensional space. J. Reine Angew. Math. 428, 161-176 (1992) Zbl 0746.52012 MR 1166511

[HHW] Hall, R. R., Hayman, W. K., Weitsman, A.W.: On asymmetry and capacity. J. Anal. Math. 56, 87-123 (1991) Zbl 0747.31004 MR 1243100

[Ka] Kawohl, B.: Rearrangements and Convexity of Level Sets in PDE. Lecture Notes in Math. 1150, Springer, Berlin (1985) Zbl 0593.35002 MR 0810619

[Li] Lions, P. L.: The concentration-compactness principle in the calculus of variations. The locally compact case. Part I. Ann. Inst. H. Poincaré Anal. Non Linéaire 1, 109-145 (1984) Zbl 0541.49009 MR 0778970

[Lo] Loiudice, A.: Improved Sobolev inequalities on the Heisenberg group. Nonlinear Anal. 62, 953-962 (2005) Zbl 1071.43005 MR 2153462

[LYZ] Lutwak, E., Yang, D., Zhang, G.: Sharp affine $L_{p}$ Sobolev inequalities. J. Differential Geom. 62, 17-38 (2002) Zbl 1073.46027 MR 1987375

[MV] Maggi, F., Villani, C.: Balls have the worst best Sobolev inequalities. J. Geom. Anal. 15, 83-121 (2005) Zbl 1086.46021 MR 2132267

[St] Struwe, M.: Variational Methods. Applications to Nonlinear Partial Differential Equations and Hamiltonian Systems. 2nd ed., Ergeb. Math. Grenzgeb. 34, Springer, Berlin (1996) Zbl 0939.49001 MR 1736116

[Ta] Talenti, G.: Best constant in Sobolev inequality. Ann. Mat. Pura Appl. 110, 353-372 (1976) Zbl 0353.46018 MR 0463908 